Article

\title{
Chemical Profiles and Protective Effect of Hedyotis diffusa Willd in Lipopolysaccharide-Induced Renal Inflammation Mice
}

\author{
Jian-Hong Ye ${ }^{1}$, Meng-Hua Liu ${ }^{2,3, *}$, Xu-Lin Zhang ${ }^{4}$ and Jing-Yu He ${ }^{4, *}$
}

Received: 7 August 2015 ; Accepted: 5 November 2015 ; Published: 13 November 2015

Academic Editor: Maurizio Battino

1 Department of Endocrinology, Foshan Hospital of Traditional Chinese Medicine, Foshan 528000, China; yejianhong2005@163.com

2 Guangdong Provincial Key Laboratory of New Drug Screening, School of Pharmaceutical Sciences, Southern Medical University, Guangzhou 510515, China

3 State Key Laboratory of Organ Failure Research, Guangdong Provincial Institute of Nephrology, Southern Medical University, Guangzhou 510515, China

4 Bioengineering Research Center, Guangzhou Institute of Advanced Technology, Chinese Academy of Sciences, Guangzhou 511458, China; zxl_lxz@live.com

* Correspondence: menghua_liu@hotmail.com (M.-H.L.); jy.he@giat.ac.cn (J.-Y.H.); Tel.: +86-20-6164-8597 (M.-H.L.); +86-20-2291-2742 (J.-Y.H.); Fax: +86-20-6164-8533 (M.-H.L.); +86-20-2291-2525 (J.-Y.H.)

\begin{abstract}
Protective effect of Hedyotis diffusa (H. diffusa) Willd against lipopolysaccharide (LPS)-induced renal inflammation was evaluated by the productions of cytokines and chemokine, and the bioactive constituents of $H$. diffusa were detected by the ultra-fast liquid chromatography -diode array detector-quadrupole-time of flight mass spectrometry (UFLC-DAD-Q-TOF-MS/MS) method. As the results showed, water extract of $H$. diffusa (equal to $5.0 \mathrm{~g} / \mathrm{kg}$ body weight) obviously protected renal tissues, significantly suppressed the productions of tumor necrosis factor- $\alpha$ (TNF- $\alpha$ ), interleukin (IL)-1 $\beta$, IL-6, and monocyte chemoattractant protein (MCP)-1, as well as significantly promoted the production of IL-10 in serum and renal tissues. According the chemical profiles of $H$. diffusa, flavonoids, iridoid glycosides and anthraquinones were greatly detected in serum from $H$. diffusa extract treatment mice. Two main chemotypes, including eight flavonoids and four iridoid glycosides were found in renal tissues from $H$. diffusa extract treatment mice. The results demonstrated that water extract of $H$. diffusa had protective effect on renal inflammation, which possibly resulted from the bioactive constituents consisting of flavonoids, iridoids and anthraquinones.
\end{abstract}

Keywords: Hedyotis diffusa Willd; chemical profiles; renal inflammation; flavonoids; iridoids; anthraquinones

\section{Introduction}

Hedyotis diffusa ( $H$. diffusa) Willd, an annual slender plant belong to Rubiaceae, is widely distributed in South of China, and other Asian country, such as Indonesia, Japan, Malaysia, Nepal, Philippines, Sri Lanka and Thailand [1]. H. diffusa named "Bai Hua She She Cao" in Chinese, is a famous traditional Chinese Medicine and applied on treating bronchitis, arthritis, rheumatism, appendicitis, sore throat, urethral infection, contusions, ulcerations and extension of malignancies [2]. Pharmacological studies show that $H$. diffusa has anticancer, anti-inflammatory, antioxidative, 
neuroprotective, hepatoprotective, anti-mutagenesis, and immunemodulating activities [2-6]. Meanwhile, phytochemical studies have shown that the major constituents of $H$. diffusa are anthraquinones, flavonoids and iridoid glycosides [7].

As known, the chemical constituents are responsible for the efficacy of herbal medicine. There are some investigations focused on traditional use of $H$. diffusa extract, the investigation on evaluating the anti-inflammatory effect and searching the anti-inflammatory constituents, especially for renal inflammation, have been rarely found during the past two decades. Clarifying which constituents absorbed into blood to produce anti-inflammatory effect plays a key role in clinical use of H. diffusa. However, the bioactive constituents are generally minor or trace after absorbed into blood. To elucidate the anti-inflammatory constituents from $H$. diffusa, the method that could improve resolution efficiency and enhance the signal response of minor/trace constituents should be used.

Ultra-fast liquid chromatography-diode array detector-quadrupole-time of flight mass spectrometry (UFLC-DAD-Q-TOF-MS/MS) is a very useful means for separation and identification of compounds from herbal extract and biological samples. The UFLC-DAD-Q-TOF-MS/MS method has been so far used to analyze the constituents in $H$. diffusa due to the high resolution and low detection limit $[8,9]$. This method, however, has not been used for research on anti-inflammatory constituents of $H$. diffusa in vivo.

In the study, the protective effect of $H$. diffusa on anti-inflammation was evaluated using histological appearance and immunohistochemistry of renal sections from lipopolysaccharide (LPS)-induced renal inflammation mice model. The levels of pro-inflammatory cytokines, anti-inflammatory cytokine and chemokine in serum and renal tissues were detected to evaluate the anti-inflammatory effect of $H$. diffusa. Furthermore, to find out the potential bioactive constituents with anti-inflammatory effect, serum and renal chemical profiles of $H$. diffusa were studied by UFLC-DAD-Q-TOF-MS/MS method.

\section{Results and Discussion}

\subsection{Effect of Water Extract of H. diffusa on the Histology of LPS-Induced Renal Inflammation Mice}

No histological changes were seen in renal section of the control group (Figure 1). In contrast, histological evaluation of renal sections from LPS-treated group revealed that necrotic epithelial cells, invasion of inflammatory cells in the interstitium and swelling glomeruli with decrease of capsular space. Comparing to LPS-treated group, the administration of low and medium doses of water extract of $H$. diffusa (1.25 and $2.5 \mathrm{~g} / \mathrm{kg}$ body weight (bw)) partially prevented renal damage induced by LPS. The high dose $(5.0 \mathrm{~g} / \mathrm{kg} \mathrm{bw})$ could have better protection to the mice renal tissue from damage induced by LPS.

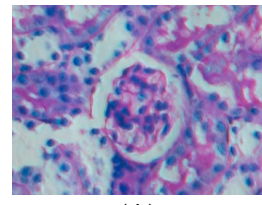

(A)

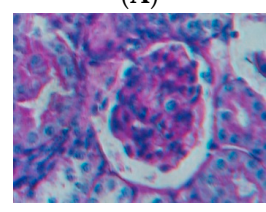

(C)

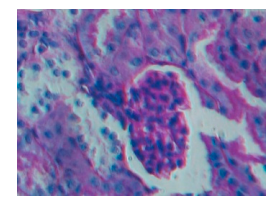

(B)

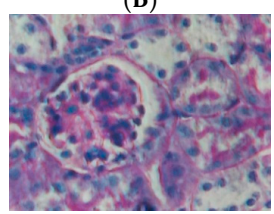

(D)

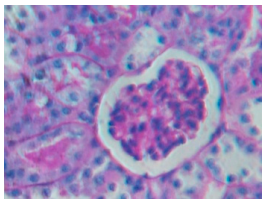

(E)

Figure 1. Effect of Hedyotis diffusa extract in the LPS-treated mice: histological appearance of renal sections of mice treated with saline (A); LPS (B); low dose (1.25 g/ $\mathrm{kg}$ body weight) (C); medium dose $(2.5 \mathrm{~g} / \mathrm{kg} \mathrm{bw})(\mathbf{D})$; and high dose $(5.0 \mathrm{~g} / \mathrm{kg} \mathrm{bw})$ (E) of water extract of $H$. diffusa. The renal sections were analyzed by PAS staining (magnification is $400 \times$ ). 
Macrophages involvement in LPS-induced renal damage was assessed, and therefore the glycoprotein CD68, one of important antigens for macrophage study, was examined after LPS-treated mice. As shown in Figure 2, CD68-positive macrophages were at low levels in control group. Compared with the after LPS-treated group, water extract of $H$. diffusa obviously reduced the CD68, indicating that the number of infiltrative macrophage was markedly inhibited after H. diffusa treatment.

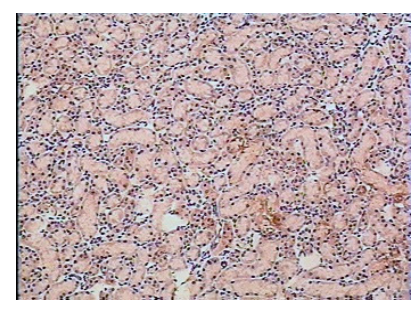

(A)

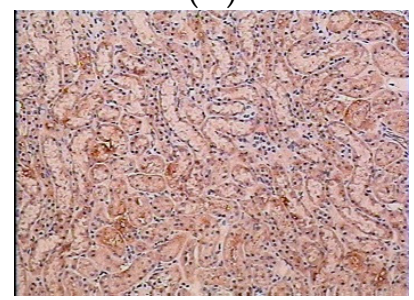

(C)

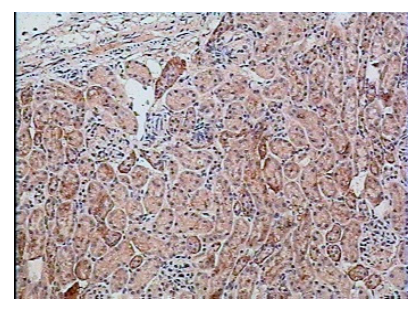

(B)

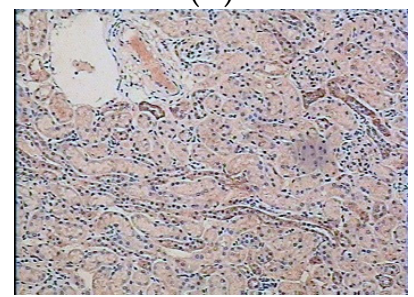

(D)

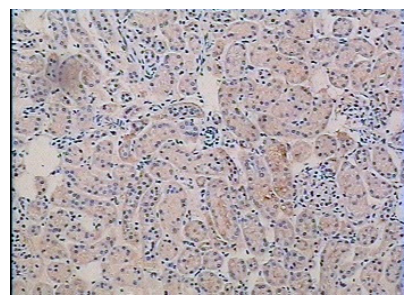

(E)

Figure 2. Effects of $H$. diffusa extract on the infiltration of macrophage in the kidneys: CD68 positive cells stained with immunohistochemistry are shown after treatment with (A) saline; (B) LPS; (C) low dose, $1.25 \mathrm{~g} / \mathrm{kg} \mathrm{bw}$; (D) medium dose, $2.5 \mathrm{~g} / \mathrm{kg}$ bw; and (E) high dose, $5.0 \mathrm{~g} / \mathrm{kg} \mathrm{bw}$ ) (magnification is $200 \times)$.

\subsection{Effect of Water Extract of H. diffusa on the Productions of Cytokines and Chemokines}

To elucidate the protective effect of $H$. diffusa on LPS-induced renal inflammation, the levels of important cytokines tumor necrosis factor- $\alpha$ (TNF- $\alpha$ ), interleukin (IL)- $1 \beta$, IL- 6 , and chemokine monocyte chemoattractant protein (MCP)-1 in serum and renal tissues were measured (Figure 3). Injection of LPS caused a significant increase in the levels of pro-inflammatory cytokines TNF- $\alpha$, IL-1 $\beta$, IL-6, and MCP-1 in serum and renal tissues and the level of anti-inflammatory cytokine IL-10 in renal tissues as compared with those in the control group. The administration of low dose of water extract of $H$. diffusa $(1.25 \mathrm{~g} / \mathrm{kg} \mathrm{bw})$ could decrease in the level of TNF- $\alpha$ in serum and renal tissues, and partially affect the factors in serum or renal tissues, including significant increase in the level of IL-10, decrease in the level of IL-6 in renal tissues and the level of MCP-1 in serum compared with that of the LPS-treated group. The medium dose treated group $(2.5 \mathrm{~g} / \mathrm{kg} \mathrm{bw})$ could significantly decrease the level of TNF- $\alpha$, IL- $1 \beta$ and IL- 6 , and increase the level of IL-10 in serum and renal tissues; moreover, this dose $(2.5 \mathrm{~g} / \mathrm{kg} \mathrm{bw})$ could also significantly decrease the level of MCP-1 in serum when compared with that of the LPS-treated group. Treatment with high dose of $H$. diffusa $(5.0 \mathrm{~g} / \mathrm{kg} \mathrm{bw})$ could significantly decrease productions of TNF- $\alpha$, IL-1 $\beta$, IL- 6 , and MCP-1, and increased production of IL-10 as compared with those in the LPS-treated group, showing the dose-dependent manner. In addition, the high dose treated group showed no significant difference to the control group in the levels of TNF- $\alpha$, IL-1 $\beta$, IL-6, and MCP-1 in serum and renal tissues, while this group had significantly higher level of IL-10 than that of the control group. 

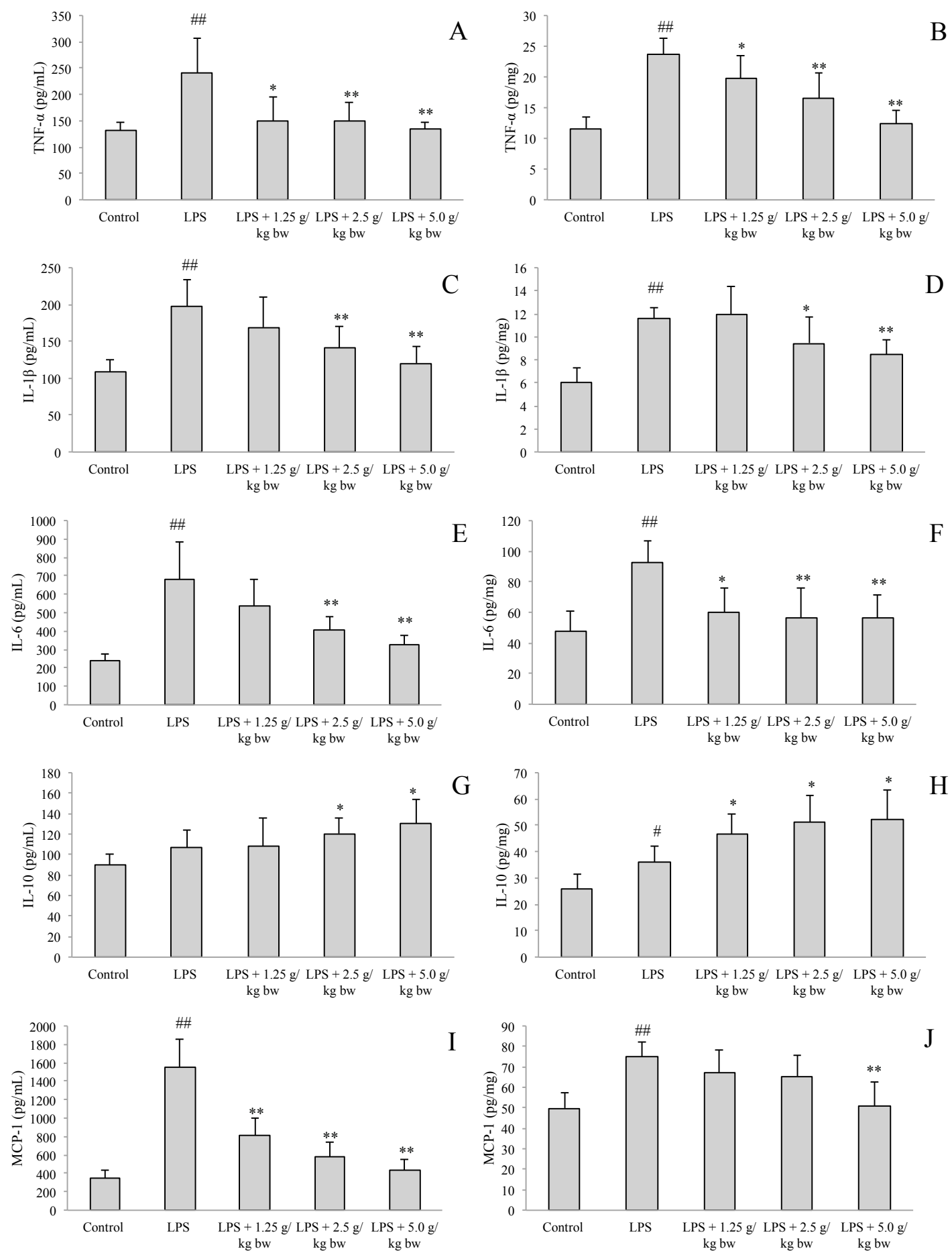

Figure 3. Effects of water extract of $H$. diffusa on the levels of TNF- $\alpha(\mathbf{A}, \mathbf{B})$, IL-1 $\beta$ (C,D), IL-6 (E,F), IL-10 (G,H), and MCP-1 (I,J) in serum and renal tissue of mice. Results are expressed as means \pm SD $(n=8) .{ }^{*} p<0.05,{ }^{* *} p<0.01$ compared with LPS group; ${ }^{\#} p<0.05,{ }^{\#} p<0.01$ compared with control group.

\subsection{Chemical Profiles of Water Extract of H. diffusa by UFLC-DAD-Q-TOF-MS/MS}

In this study, water extract of $H$. diffusa was detected both in positive ion mode and negative ion mode (Figure 4). The detailed information of 35 compounds has been listed in Table 1 and their chemical structures were shown in Figure 5. Simple peaks 1, 2, 3, 4, 5, 6, 7, 8, 11, 15, 24, 26 
and 35 have unequivocally identified as deacetylasperulosidec acid, scandoside, geniposidic acid, scandoside methyl ester, asperulosidic acid, scopolin, asperuloside, $p$-courmaric acid, ferulic acid, rutin, quercetin, kaemperol and ursolic acid, respectively, by comparing their retention time, UV data, molecular weight and MS spectra with those of standards. The constituents that were with no referable standards have been tentatively characterized with UV data, molecular weight, and fragment behaviors in MS spectra and from the literature.
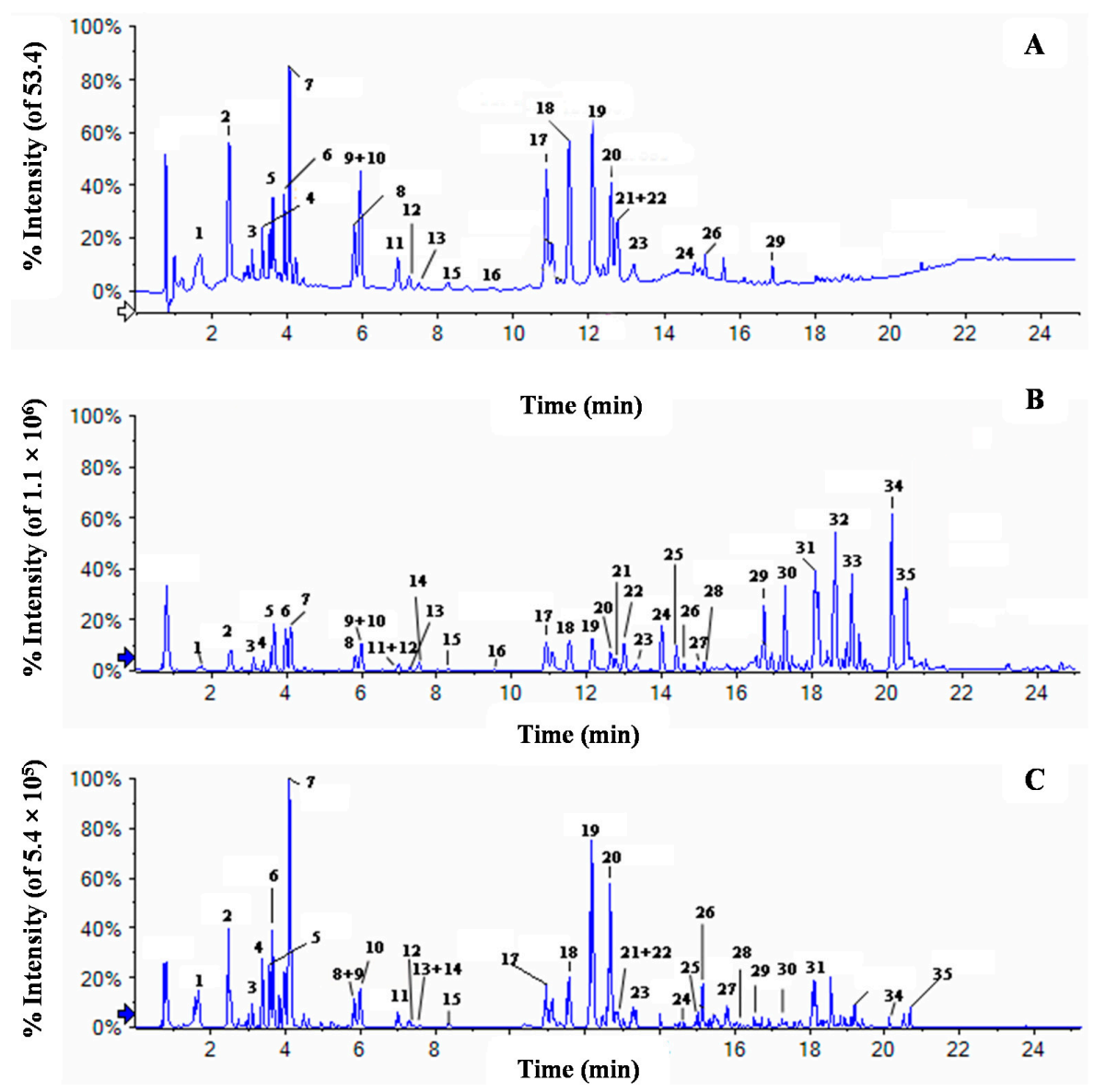

Figure 4. Chromatograms of water extract of H. diffusa: (A) DAD chromatogram detected at $254 \mathrm{~nm}$; (B) total ion chromatogram in positive ion mode; and (C) total ion chromatogram in negative ion mode). White arrow means DAD chromatogram (A) and the blue arrows means MS chromatogram in positive and negative ion mode $(\mathbf{B}, \mathbf{C})$.

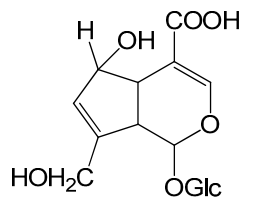

Deacetylasperulosidic acid (1)<smiles>CC(=O)C1=COC(OCl)C2C(CO)=CC(O)C12</smiles>

Scandoside methyl ester (4)

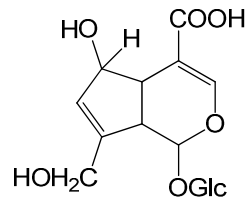

Scandoside (2)

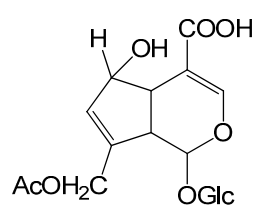

Asperulosidic acid (5)

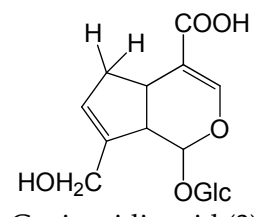

Geniposidic acid (3)<smiles>COc1cc2ccc(=O)oc2cc1OC</smiles>

Scopolin (6)

Figure 5. Cont. 
<smiles>CC(=O)OCC1=CC2OC(=O)C3C=C(OC(C)=O)C(O1)C23</smiles>

Asperuloside (7)
$\mathrm{HO}$

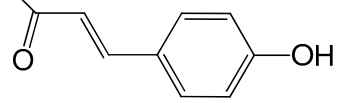

$p$-Coumaric acid (8)

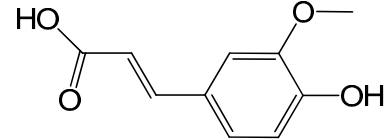

Ferulic acid (11)<smiles>[R]Oc1c(-c2ccc(O)c(O)c2)oc2cc(O)cc(O)c2c1=O</smiles>

Quercetin-3-O-sophoroside (9): R = glc-(1"'-2"')-glc; Quercetin-3-O-[2-O- $\beta$-D-glucopyranosyl]- $\beta$-Dgalactopyranoside (10): R = glc-(1"'-2'"')-gal; Quercetin-3-O-sambubioside (12): R = xyl-(1"'-2"')-glc; Rutin (15): $\mathrm{R}=$ rutinoside; Quercetin-3-O-[2-O-(6-O-E-sinapol)- $\beta$-D-glucopyranosyl]- $\beta$-D-galactopyranoside (17): $\mathrm{R}=66^{\prime \prime \prime}-$

$O$-sinapoyl-glc- (1"'-2"')-gal; Quercetin-3-O-[2-O-(6-O-E- feruloyl)- $\beta$-D-glucopyranosyl]- $\beta$-Dgalactopyranoside (18): $\mathrm{R}=6^{\prime \prime \prime}-\mathrm{O}$-feruloyl-glc- (1"'-2"')-gal; Quercetin (24): $\mathrm{R}=\mathrm{H}$<smiles>[R]c1c(-c2ccc(O)cc2)oc2cc(O)cc(O)c2c1=O</smiles>

Kaempferol-3-O-(2-O- $\beta$-D-glucopyranosyl)- $\beta$-D-galactopyranoside (13/14): R=glc-(1"'-2'"')-gal; Kaempferol-3-O-(6"-O-L-rhamnosyl)- $\beta$-D-glucopyranoside (16) $\mathrm{R}$ = rha-(1"'-6"')-glc; Kaempferol-3-O-[2-O(6-O-E-feruloyl)- $\beta$-D-glucopyranosyl]- $\beta$-D-galactopyranoside (21): $\mathrm{R}=6^{\prime \prime \prime}-\mathrm{O}$-feruloy-glc-(1"'-2'"')-gal;

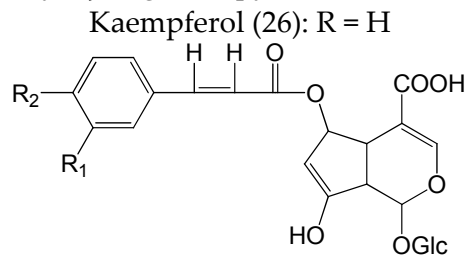

6-O-Z-p-coumaroyl scandoside methyl ester (19): $\mathrm{R}_{1}=\mathrm{OH}, \mathrm{R}_{2}=\mathrm{H}$; 6-O-Z-p-feruloyl scandoside methyl ester (22): $\mathrm{R}_{1}=\mathrm{OCH}_{3}, \mathrm{R}_{2}=\mathrm{OH}$<smiles>[R]c1ccc(/C=C/C(=O)OC2C=C(O)C3C(OC)OC=C(O)C23)cc1[R]</smiles>

6-O-E-p-coumaroyl scandoside methyl ester (20): $\mathrm{R}_{1}=\mathrm{OH}, \mathrm{R}_{2}=\mathrm{H}$; 6-O-E- $p$-feruloyl scandoside methyl ester (23): $\mathrm{R}_{1}=\mathrm{OCH}_{3}, \mathrm{R}_{2}=\mathrm{OH}$<smiles>COc1c(O)ccc2c1C(=O)c1ccccc1C2=O</smiles><smiles>COc1c(C)c(O)cc2c1C(=O)c1cc(O)ccc1C2=O</smiles>

1-Methoxy-2-hydroxyanthraquinone (27) 2,6-Dihydroxy-3-methyl-4-methoxyanthraquinone (25/28/30)<smiles>COc1c(O)c(C)cc2c1C(=O)c1ccccc1C2=O</smiles>

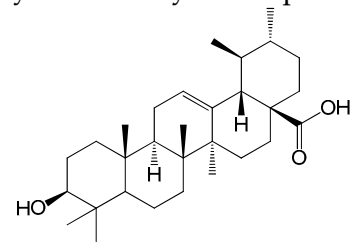

1-Methoxy-2-hydroxy-3-methylanthraquinone (29)

Figure 5. Chemical structures of the compounds identified in H. diffusa extract, mice plasma and kidney. 
Due to abundant capillary in mice abdomen, constituents in H. diffusa could be absorbed quickly and comprehensively by intraperitoneal injection. After deducting the matrix interference from control plasma and model plasma, most of these compounds, except peaks $6,22,23,33$ and 35, were also found in dosed plasma. However, only peaks 1, 2, 3, 4, 9, 10, 13, 14, 16, 18, 24, 26 and 27 were detected in kidney homogenate by XIC manager (Extract Ions Using Dialog). Peaks 31, 32, and 34 were found in bio-samples from control and model group as well as from dosed group. In view of the fragmentor rule, they were inferred as polyunsaturated fats compounds. The tentative identification of each components were outlined below.

6-O-Z-p-Coumaroyl scandoside methyl ester (peak 19) and 6-O-E-p-coumaroyl scandoside methyl ester (peak 20) were isomers with $[\mathrm{M}-\mathrm{H}]^{-}$ions at $\mathrm{m} / z 549.16274$ and $\mathrm{m} / z 549.16396$ $\left(\mathrm{C}_{26} \mathrm{H}_{30} \mathrm{O}_{13}\right)$, respectively, in negative ion mode. Peaks 22 and 23 were identified as 6-O-Z-p-feruloyl scandoside methyl ester and 6-O-E-p-feruloyl scandoside methyl ester, $30 \mathrm{Da}$ more than peak 19 . The produced fragment ion at $m / z 223, m / z 193$, and $m / z 119$ from these four compounds were similar to scandoside methyl ester. It indicated that the four compounds were the derivatives of scandoside methyl ester, which is in accordance with previous literature [8,9].

Flavonoids and its derivatives showed characteristic UV spectra and fragment rules in mass spectra under collision induced decomposition condition [10,11]. The groups linked to aglycone, such as glucose, galactose and rhamnose, were always loss firstly and the deprotonated aglycone always had a high relative aboundance. Compounds 9 and 10 were a pair of isomers with the protonated molecular ions at $m / z 627.15557$ and $m / z 627.15525\left(\mathrm{C}_{27} \mathrm{H}_{30} \mathrm{O}_{17}\right)$, respectively, and similar fragmentations, showing the occurrence of two hexosyl residue. Referring to the literature [12], they were tentatively identified as quercetin-3-O-sophoroside and quercetin-3-O-[2-O- $\beta$-D-glucopyranosyl]- $\beta$-D-galactopyranoside. Compound 12 gave protonated ion at $m / z 597.14508\left(\mathrm{C}_{26} \mathrm{H}_{28} \mathrm{O}_{16}\right)$. The MS/MS analyses showed the loss of $132 \mathrm{Da}$ and $294 \mathrm{Da}$ $(132+162)$, suggesting the presence of xylose and glucose groups. Therefore, it was identified as quercetin-3-O-sambubioside in according with previous report [13]. Compound 17 was characterized as quercetin-3-O-[2-O-(6-O-E-sinapol)- $\beta$-D-glucopyranosyl]- $\beta$-D-galactopyranoside with deprotonated molecular ion at $m / z 831.19906\left(\mathrm{C}_{38} \mathrm{H}_{40} \mathrm{O}_{21}\right), 206$ Da higher than that of compound 10 , suggesting that it was a sinapol derivated of $10[9,13]$. In addition, the base peak at $\mathrm{m} / z 301.0344$ demonstrated the presence of quercetin. Compound 18 gave deprodonated molecular ion at $\mathrm{m} / z$ $801.18894\left(\mathrm{C}_{37} \mathrm{H}_{38} \mathrm{O}_{20}\right)$, with similar MS/MS spectra with compound 17. It was 30 Da less than that of compound 17, indicating the lack of a methoxyl group. Thus, it was tentatively characterized as quercetin-3-O-[2-O-(6-O-E-feruloyl)- $\beta$-D-glucopyranosyl]- $\beta$-D-galactopyranoside [12]. Compounds $13,14,16$ and 21 showed the characteristic MS fragmentation of kaempferol derivated. The loss of 324 $\mathrm{Da}(162+162)$ for 13 and $14,306 \mathrm{Da}(146+162)$ for 16 and $500 \mathrm{Da}(176+162+162)$ indicated different groups linked to kaempferol aglycone. Because of the lack of more evidence, compounds 13 and 14 were tentatively characterized as Kaempferol-3-O-(2-O- $\beta$-D-glucopyranosyl)- $\beta$-D-galactopyranoside and its isomer [12,14], and need to be confirmed by NMR. Compounds 16 and 21 were tentatively characterized as kaempferol-3-O-(6"-O-L-rhamnosyl)- $\beta$-D-glucopyranoside and kaempferol-3-O-[2-O-(6-O-E-feruloyl)- $\beta$-D-glucopyranosyl]- $\beta$-D-galactopyranoside, respectively [12,15]. 
Table 1. Chemical profiles in $H$ diffusa by ultra-fast liquid chromatography-diode array detector-quadrupole-time of flight mass spectrometry.

\begin{tabular}{|c|c|c|c|c|c|c|c|c|c|c|}
\hline No. & Rt (min) & $\begin{array}{l}\text { Molecular } \\
\text { Formula } \\
\end{array}$ & $\begin{array}{l}\lambda \max \\
(\mathrm{nm})\end{array}$ & {$[\mathrm{M}+\mathrm{Na}]^{+}$} & {$[\mathbf{M}+\mathbf{H}]^{+}$} & {$[\mathbf{M}-\mathbf{H}]^{-}$} & $\begin{array}{l}\text { Major Fragmentors in } \\
\text { Positive Mode }\end{array}$ & $\begin{array}{l}\text { Major Fragmentors in } \\
\text { Negative Mode }\end{array}$ & Identification & Source \\
\hline 1 & 1.62 & $\mathrm{C}_{16} \mathrm{H}_{22} \mathrm{O}_{11}$ & 239 & $\begin{array}{l}413.10505 \\
(-0.9)\end{array}$ & & $389.10862(-0.8)$ & $\begin{array}{l}395.0879\left[\mathrm{M}+\mathrm{Na}-\mathrm{H}_{2} \mathrm{O}\right]^{+}, \\
251.0512\left[\mathrm{M}+\mathrm{Na}-\mathrm{glc}^{+},\right. \\
233.0420\left[\mathrm{M}+\mathrm{H}-\mathrm{glc}^{+},\right. \\
215.0299 \\
{\left[\mathrm{M}+\mathrm{H}-\text { glc- } \mathrm{H}_{2} \mathrm{O}\right]^{+}}\end{array}$ & $\begin{array}{l}227.0579\left[\mathrm{M}-\mathrm{H}-\mathrm{glc}^{-}, 209.0468\right. \\
{\left[\mathrm{M}-\mathrm{H}-\mathrm{glc}-\mathrm{H}_{2} \mathrm{O}\right]^{-}, 183.0683} \\
{\left[\mathrm{M}-\mathrm{H}-\mathrm{glc}-\mathrm{CO}_{2}\right]^{-}, 165.0573} \\
{\left[\mathrm{M}-\mathrm{H}-\mathrm{glc}-\mathrm{CO}_{2}-\mathrm{H}_{2} \mathrm{O}\right]^{-}, 137.0616} \\
{\left[\mathrm{M}-\mathrm{H}-\text { glc- }-\mathrm{CO}_{2}-\mathrm{H}_{2} \mathrm{O}-\mathrm{CO}\right]^{-}}\end{array}$ & $\begin{array}{l}\text { Deacetylasperulosidic } \\
\text { acid }\end{array}$ & $\begin{array}{l}\text { Herb, } \\
\text { Plasma, } \\
\text { Kidney }\end{array}$ \\
\hline 2 & 2.50 & $\mathrm{C}_{16} \mathrm{H}_{22} \mathrm{O}_{11}$ & 238 & $\begin{array}{l}413.10505 \\
(-0.9)\end{array}$ & & $\begin{array}{l}389.10862 \\
(-0.8)\end{array}$ & $\begin{array}{l}395.0948\left[\mathrm{M}+\mathrm{Na}-\mathrm{H}_{2} \mathrm{O}\right]^{+} \\
233.0408\left[\mathrm{M}+\mathrm{H}-\mathrm{glc}^{+}\right.\end{array}$ & 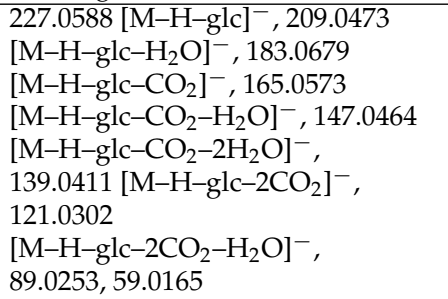 & Scandoside & $\begin{array}{l}\text { Herb, } \\
\text { Plasma, } \\
\text { Kidney }\end{array}$ \\
\hline 3 & 3.01 & $\mathrm{C}_{16} \mathrm{H}_{22} \mathrm{O}_{10}$ & 235 & $397.11053(+0)$ & & $\begin{array}{l}373.11388 \\
(-0.4)\end{array}$ & $\begin{array}{l}235.0561[\mathrm{M}+\mathrm{Na}-\mathrm{glc}]^{+}, \\
217.0439,191.0673\end{array}$ & $\begin{array}{l}211.0620[\mathrm{M}-\mathrm{H}-\mathrm{glc}]^{-}, 167.0707 \\
{\left[\mathrm{M}-\mathrm{H}-\mathrm{glc}-\mathrm{CO}_{2}\right]^{-}, 149.0606} \\
123.0453\end{array}$ & Geniposidic acid & $\begin{array}{l}\text { Herb, } \\
\text { Plasma, } \\
\text { Kidney }\end{array}$ \\
\hline 4 & 3.38 & $\mathrm{C}_{17} \mathrm{H}_{24} \mathrm{O}_{11}$ & 238 & $\begin{array}{l}427.12095 \\
(-0.3)\end{array}$ & & $\begin{array}{l}403.12433 \\
(-0.6)\end{array}$ & $265.0669[\mathrm{M}+\mathrm{Na}-\mathrm{glc}]^{+}$ & $\begin{array}{l}241.0710[\mathrm{M}-\mathrm{H}-\mathrm{glc}]^{-}, 223.0628 \\
\left.\mathrm{IM}-\mathrm{H}-\text { glc- }_{2} \mathrm{O}\right]^{-}, 209.0444 \\
{[\mathrm{M}-\mathrm{H}-\text { glc-CH }} \\
191.0508,167.0350,139.0397\end{array}$ & $\begin{array}{l}\text { Scandoside methyl } \\
\text { ester }\end{array}$ & $\begin{array}{l}\text { Herb, } \\
\text { Plasma, } \\
\text { Kidney }\end{array}$ \\
\hline 5 & 3.60 & $\mathrm{C}_{18} \mathrm{H}_{24} \mathrm{O}_{12}$ & 229 & $\begin{array}{l}455.11562 \\
(-0.8)\end{array}$ & & $\begin{array}{l}431.11967 \\
(+0.4)\end{array}$ & $\begin{array}{l}437.1027\left[\mathrm{M}+\mathrm{Na}-\mathrm{H}_{2} \mathrm{O}\right]^{+}, \\
293.0621\left[\mathrm{M}+\mathrm{Na}-\mathrm{glc}^{+},\right. \\
275.0518 \\
{\left[\mathrm{M}+\mathrm{H}-\mathrm{glc}-\mathrm{H}_{2} \mathrm{O}\right]^{+}} \\
197.0191,147.0430\end{array}$ & $\begin{array}{l}269.0669[\mathrm{M}-\mathrm{H}-\text { glc }]^{-}, 251.0588 \\
{\left[\mathrm{M}-\mathrm{H}-\text { glc- } \mathrm{H}_{2} \mathrm{O}\right]^{-}, 225.0792} \\
{\left[\mathrm{M}-\mathrm{H}-\text { glc- } \mathrm{CO}_{2}\right]^{-}, 179.0566} \\
{\left[\mathrm{M}-\mathrm{H}-\text { glc- } \mathrm{CO}_{2}-\mathrm{HCOOH}\right]^{-},} \\
165.0573 \\
{\left[\mathrm{M}-\mathrm{H}-\mathrm{glc}-\mathrm{CO}_{2}-\mathrm{CH}_{3} \mathrm{COOH}\right]^{-},} \\
121.0302 \\
{\left[\mathrm{M}-\mathrm{H}-\mathrm{glc}-2 \mathrm{CO}_{2}-\mathrm{CH}_{3} \mathrm{COOH}\right]^{-},} \\
89.0257,59.0170\end{array}$ & Asperulosidic acid & $\begin{array}{l}\text { Herb, } \\
\text { Plasma }\end{array}$ \\
\hline 6 & 3.98 & $\mathrm{C}_{16} \mathrm{H}_{18} \mathrm{O}_{9}$ & $\begin{array}{l}221, \\
320\end{array}$ & & $\begin{array}{l}355.10270 \\
(+1.0)\end{array}$ & $\begin{array}{l}353.0881 \\
(+0.8)\end{array}$ & $\begin{array}{l}163.0384,145.0238, \\
135.0442,117.0343, \\
89.0398\end{array}$ & $\begin{array}{l}\text { 191.0552 [M-H-glc }]^{-}, 179.0341, \\
135.0446\end{array}$ & Scopolin & Herb \\
\hline 7 & 4.13 & $\mathrm{C}_{18} \mathrm{H}_{22} \mathrm{O}_{11}$ & 238 & $\begin{array}{l}437.10509 \\
(-0.3)\end{array}$ & & $\begin{array}{l}413.1085 \\
(-0.9)\end{array}$ & $\begin{array}{l}275.0542[\mathrm{M}+\mathrm{Na}-\mathrm{glc}]^{+} \\
187.0372,147.0458\end{array}$ & $\begin{array}{l}251.0544[\mathrm{M}-\mathrm{H}-\mathrm{glc}]^{-}, 205.0529 \\
{[\mathrm{M}-\mathrm{H}-\text { glc-HCOOH}]^{-}, 191.0364} \\
{\left[\mathrm{M}-\mathrm{H}-\text { glc- } \mathrm{CH}_{3} \mathrm{COOH}\right]^{-},} \\
147.0457 \\
{\left[\mathrm{M}-\mathrm{H}-\text { glc- } \mathrm{CH}_{3} \mathrm{COOH}-\mathrm{CO}_{2}\right]^{-},} \\
119.0501 \\
{\left[\mathrm{M}-\mathrm{H}-\mathrm{glc}-\mathrm{CH}_{3} \mathrm{COOH}-\mathrm{CO}-\mathrm{CO}_{2}\right]^{-}}\end{array}$ & Asperuloside & $\begin{array}{l}\text { Herb, } \\
\text { Plasma }\end{array}$ \\
\hline
\end{tabular}


Table 1. Cont.

\begin{tabular}{|c|c|c|c|c|c|c|c|c|c|c|}
\hline No. & Rt (min) & $\begin{array}{l}\text { Molecular } \\
\text { Formula } \\
\end{array}$ & $\begin{array}{l}\lambda \max \\
(\mathrm{nm})\end{array}$ & {$[\mathrm{M}+\mathrm{Na}]^{+}$} & {$[\mathbf{M}+\mathbf{H}]^{+}$} & {$[\mathbf{M}-\mathbf{H}]^{-}$} & $\begin{array}{l}\text { Major Fragmentors in } \\
\text { Positive Mode }\end{array}$ & $\begin{array}{l}\text { Major Fragmentors in } \\
\text { Negative Mode }\end{array}$ & Identification & Source \\
\hline 8 & 5.79 & $\mathrm{C}_{9} \mathrm{H}_{8} \mathrm{O}_{3}$ & $\begin{array}{l}219 \\
296\end{array}$ & & $\begin{array}{l}165.05416 \\
(-2.8)\end{array}$ & $\begin{array}{l}163.04128 \\
(7.4)\end{array}$ & $\begin{array}{l}147.0435\left[\mathrm{M}+\mathrm{H}-\mathrm{H}_{2} \mathrm{O}\right]^{+} \\
119.049 \\
{[\mathrm{M}+\mathrm{H}-\mathrm{HCOOH}]^{+}} \\
91.0552,77.0406,65.0415\end{array}$ & $\begin{array}{l}119.0505\left[\mathrm{M}-\mathrm{H}-\mathrm{CO}_{2}\right]^{-} \\
93.0355\end{array}$ & $p$-Coumaric acid & $\begin{array}{l}\text { Herb, } \\
\text { Plasma }\end{array}$ \\
\hline 9 & 5.85 & $\mathrm{C}_{27} \mathrm{H}_{30} \mathrm{O}_{17}$ & $\begin{array}{l}256 \\
355\end{array}$ & $\begin{array}{l}649.1370 \\
(-0.8)\end{array}$ & $\begin{array}{l}627.15557 \\
(+)\end{array}$ & $\begin{array}{l}625.14120 \\
(+0.3)\end{array}$ & $\begin{array}{l}465.1052[\mathrm{M}+\mathrm{H}-\mathrm{glc}]^{+}, \\
303.0490[\mathrm{M}+\mathrm{H}-2 \mathrm{glc}]^{+}\end{array}$ & 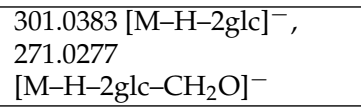 & $\begin{array}{l}\text { Quercetin-3-O- } \\
\text { sophoroside }\end{array}$ & $\begin{array}{l}\text { Herb, } \\
\text { Plasma, } \\
\text { Kidney }\end{array}$ \\
\hline 10 & 5.99 & $\mathrm{C}_{27} \mathrm{H}_{30} \mathrm{O}_{17}$ & $\begin{array}{l}256 \\
355\end{array}$ & $649.1370(-0.8)$ & $\begin{array}{l}627.15525 \\
(-0.5)\end{array}$ & & $\begin{array}{l}465.1021\left[\mathrm{M}+\mathrm{H}-\text { gal }^{+},\right. \\
303.0493 \\
\text { [M+H-gal-glc }^{+}\end{array}$ & $\begin{array}{l}301.0317[\mathrm{M}-\mathrm{H}-\mathrm{gal}-\mathrm{glc}]^{-} \\
271.0280 \\
{[\mathrm{M}-\mathrm{H}-\mathrm{gal}-\mathrm{glc}-\mathrm{HCOH}]^{-}}\end{array}$ & $\begin{array}{l}\text { Quercetin-3-O-[2-O- } \beta- \\
\text { D-glucopyranosyl]- } \beta \text {-D- } \\
\text { galactopyranoside }\end{array}$ & $\begin{array}{l}\text { Herb, } \\
\text { Plasma, } \\
\text { Kidney }\end{array}$ \\
\hline 11 & 6.92 & $\mathrm{C}_{10} \mathrm{H}_{10} \mathrm{O}_{4}$ & 219 & & $\begin{array}{l}195.06477 \\
(-2.1)\end{array}$ & $\begin{array}{l}193.05146 \\
(+4.3)\end{array}$ & $\begin{array}{l}177.0549\left[\mathrm{M}+\mathrm{H}-\mathrm{H}_{2} \mathrm{O}\right]^{+} \\
149.0523 \\
{[\mathrm{M}+\mathrm{H}-\mathrm{HCOOH}]^{+}} \\
145.0283,89.0396\end{array}$ & $149.0457\left[\mathrm{M}-\mathrm{H}-\mathrm{CO}_{2}\right]^{-}$ & Ferulic acid & $\begin{array}{l}\text { Herb, } \\
\text { Plasma }\end{array}$ \\
\hline 12 & 7.01 & $\mathrm{C}_{26} \mathrm{H}_{28} \mathrm{O}_{16}$ & $\begin{array}{l}264 \\
339\end{array}$ & $\begin{array}{l}619.12649 \\
(-0.8)\end{array}$ & $\begin{array}{l}597.14508 \\
(+0.1)\end{array}$ & $\begin{array}{l}595.13022 \\
(-0.4)\end{array}$ & $\begin{array}{l}465.1011[\mathrm{M}+\mathrm{H}-\mathrm{xyl}]^{+}, \\
303.0484 \\
{[\mathrm{M}+\mathrm{H}-\mathrm{xyl}-\mathrm{glc}]^{+}}\end{array}$ & 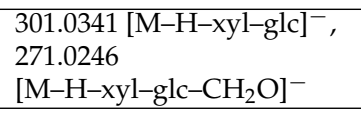 & $\begin{array}{l}\text { Quercetin-3- } \\
\text { O-sambubioside }\end{array}$ & $\begin{array}{l}\text { Herb, } \\
\text { Plasma }\end{array}$ \\
\hline 13 & 7.27 & $\mathrm{C}_{27} \mathrm{H}_{30} \mathrm{O}_{16}$ & $\begin{array}{l}265 \\
344\end{array}$ & $\begin{array}{l}633.14211 \\
(-0.8)\end{array}$ & $\begin{array}{l}611.16075 \\
(+0.1)\end{array}$ & $\begin{array}{l}609.14959 \\
(+5.6)\end{array}$ & $\begin{array}{l}449.1062[\mathrm{M}+\mathrm{H}-\mathrm{glc}]^{+}, \\
287.0540 \\
{[\mathrm{M}+\mathrm{H}-\text { glc-gal }]^{+},} \\
163.0598\end{array}$ & $\begin{array}{l}285.0348 \text { [M-H-glc-gal] }^{-}, \\
255.0320 \\
\left.{ } \mathrm{M}^{-} \mathrm{H}-\text { glc-gal-}-\mathrm{CH}_{2} \mathrm{O}\right]^{-}\end{array}$ & $\begin{array}{l}\text { Kaempferol-3-O-(2-O- } \beta \text { - } \\
\text { D-glucopyranosyl)- } \beta \text {-D- } \\
\text { galactopyranoside or } \\
\text { isomer }\end{array}$ & $\begin{array}{l}\text { Herb, } \\
\text { Plasma, } \\
\text { Kidney }\end{array}$ \\
\hline 14 & 7.57 & $\mathrm{C}_{27} \mathrm{H}_{30} \mathrm{O}_{16}$ & $\begin{array}{l}264 \\
340\end{array}$ & $\begin{array}{l}633.14211 \\
(-0.8)\end{array}$ & $\begin{array}{l}611.16069 \\
(0)\end{array}$ & $\begin{array}{l}609.15134 \\
(+8.6)\end{array}$ & $\begin{array}{l}449.1081\left[\mathrm{M}+\mathrm{H}-\mathrm{glc}^{+},\right. \\
287.0541 \\
{[\mathrm{M}+\mathrm{H}-\mathrm{glc}-\mathrm{gal}]^{+},} \\
145.0482\end{array}$ & $\begin{array}{l}285.0434 \text { [M-H-glc-gal }^{-} \\
255.0320 \\
\left.{ }^{-} \mathrm{M}-\mathrm{H}-\text { glc-gal- } \mathrm{CH}_{2} \mathrm{O}\right]^{-}\end{array}$ & $\begin{array}{l}\text { Kaempferol-3-O-(2-O- } \beta- \\
\text { D-glucopyranosyl)- } \beta \text {-D- } \\
\text { galactopyranoside or } \\
\text { isomer }\end{array}$ & $\begin{array}{l}\text { Herb, } \\
\text { Plasma, } \\
\text { Kidney }\end{array}$ \\
\hline 15 & 8.31 & $\mathrm{C}_{27} \mathrm{H}_{30} \mathrm{O}_{16}$ & $\begin{array}{l}265 \\
340\end{array}$ & $\begin{array}{l}633.14211 \\
(-0.8)\end{array}$ & $\begin{array}{l}611.16069 \\
(0)\end{array}$ & $\begin{array}{l}609.15128 \\
(+8.5)\end{array}$ & $\begin{array}{l}465.1016[\mathrm{M}+\mathrm{H}-\mathrm{rha}]^{+}, \\
303.0489 \\
{[\mathrm{M}+\mathrm{H}-\mathrm{rha}-\mathrm{glc}]^{+}}\end{array}$ & 301.0377 [M-H-rha-glc] $]^{-}$ & Rutin & $\begin{array}{l}\text { Herb, } \\
\text { Plasma }\end{array}$ \\
\hline 16 & 9.39 & $\mathrm{C}_{27} \mathrm{H}_{30} \mathrm{O}_{15}$ & $\begin{array}{l}265 \\
340\end{array}$ & & $\begin{array}{l}595.16572 \\
(0)\end{array}$ & $\begin{array}{l}593.15110 \\
(-0.2)\end{array}$ & $\begin{array}{l}449.1026[\mathrm{M}+\mathrm{H}-\mathrm{rha}]^{+}, \\
287.0548 \\
{[\mathrm{M}+\mathrm{H}-\mathrm{rha}-\mathrm{glc}]^{+}}\end{array}$ & 285.0459 [M-H-rha-glc] ${ }^{-}$ & $\begin{array}{l}\text { Kaempferol-3-O-(6" }{ }^{\prime \prime}-\mathrm{O}-\mathrm{L}- \\
\text { rhamnosyl)- } \beta \text {-D- } \\
\text { glucopyranoside }\end{array}$ & $\begin{array}{l}\text { Herb, } \\
\text { Plasma, } \\
\text { Kidney }\end{array}$ \\
\hline 17 & 10.92 & $\mathrm{C}_{38} \mathrm{H}_{40} \mathrm{O}_{21}$ & $\begin{array}{l}265 \\
338\end{array}$ & $\begin{array}{l}855.19472 \\
(-0.8)\end{array}$ & $\begin{array}{l}833.21331 \\
(-0.2)\end{array}$ & $\begin{array}{l}831.19906 \\
(+0.2)\end{array}$ & $\begin{array}{l}465.1032303 .0499 \\
{[\mathrm{M}+\mathrm{H}-\text { sinapol-glc }]^{+}} \\
303.0499 \\
{[\mathrm{M}+\mathrm{H}-\text {-sinapol-glc-gal }]^{+}} \\
177.0489\end{array}$ & $\begin{array}{l}625.1414 \text { [M-H-sinapol] }{ }^{-} \\
301.0344 \\
{\text { [M-H-sinapol-glc-gal }{ }^{-}}, \\
271.0250\end{array}$ & $\begin{array}{l}\text { Quercetin-3-O-[2-O-(6-O- } \\
\text { E-sinapol)- } \beta \text {-D- } \\
\text { glucopyranosyl]- } \beta \text {-D- } \\
\text { galactopyranoside }\end{array}$ & $\begin{array}{l}\text { Herb, } \\
\text { Plasma }\end{array}$ \\
\hline 18 & 11.54 & $\mathrm{C}_{37} \mathrm{H}_{38} \mathrm{O}_{20}$ & $\begin{array}{l}254 \\
336\end{array}$ & $\begin{array}{l}825.18454 \\
(-0.4)\end{array}$ & $\begin{array}{l}803.20329 \\
(+0.2)\end{array}$ & $\begin{array}{l}801.18894 \\
(+0.7)\end{array}$ & $\begin{array}{l}303.0493 \\
{[\mathrm{M}+\mathrm{H}-\text { feruloyl-glc-gal }]^{+}} \\
177.0539\end{array}$ & 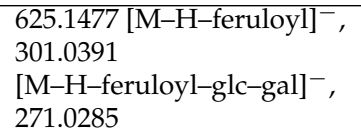 & $\begin{array}{l}\text { Quercetin-3-O-[2-O-(6-O- } \\
E \text {-feruloyl)- } \beta \text {-D- } \\
\text { glucopyranosyl]- } \beta \text {-D- } \\
\text { galactopyranoside }\end{array}$ & $\begin{array}{l}\text { Herb, } \\
\text { Plasma, } \\
\text { Kidney }\end{array}$ \\
\hline
\end{tabular}


Table 1. Cont.

\begin{tabular}{|c|c|c|c|c|c|c|c|c|c|c|}
\hline No. & Rt (min) & $\begin{array}{l}\text { Molecular } \\
\text { Formula } \\
\end{array}$ & $\begin{array}{l}\lambda \max \\
(\mathrm{nm})\end{array}$ & {$[\mathrm{M}+\mathrm{Na}]^{+}$} & {$[\mathrm{M}+\mathrm{H}]^{+}$} & {$[\mathbf{M}-\mathbf{H}]^{-}$} & $\begin{array}{l}\text { Major Fragmentors in } \\
\text { Positive Mode }\end{array}$ & $\begin{array}{l}\text { Major Fragmentors in } \\
\text { Negative Mode }\end{array}$ & Identification & Source \\
\hline 19 & 12.16 & $\mathrm{C}_{26} \mathrm{H}_{30} \mathrm{O}_{13}$ & 309 & $573.15785(+0)$ & $\begin{array}{l}551.17641 \\
(+0.9)\end{array}$ & $\begin{array}{l}549.16274 \\
(+2.5)\end{array}$ & & $\begin{array}{l}\left.\text { 369.1011 [M-H-glc- } \mathrm{H}_{2} \mathrm{O}\right]^{-}, \\
\left.\text {223.0634 [M-H-glc- } \mathrm{H}_{2} \mathrm{O}-\mathrm{cou}\right]^{-}, \\
\text {193.0552, } 191.0369,163.0409, \\
119.0519\end{array}$ & $\begin{array}{l}\text { 6-O-Z-p-coumaroyl } \\
\text { scandoside methyl } \\
\text { ester }\end{array}$ & $\begin{array}{l}\text { Herb, } \\
\text { Plasma }\end{array}$ \\
\hline 20 & 12.64 & $\mathrm{C}_{26} \mathrm{H}_{30} \mathrm{O}_{13}$ & 309 & $573.15785(+0)$ & $\begin{array}{l}551.17589 \\
(0)\end{array}$ & $\begin{array}{l}549.16396 \\
(+4.7)\end{array}$ & $\begin{array}{l}225.0723 \\
{\left[_{\left.\mathrm{M}+\mathrm{H}-\text { glc- }-\mathrm{H}_{2} \mathrm{O}-\mathrm{cou}\right]^{+}}\right.}\end{array}$ & $\begin{array}{l}\left.\text { 369.0978 [M-H-glc- } \mathrm{H}_{2} \mathrm{O}\right]^{-}, \\
\text {223.0616 }\left[\mathrm{M}-\mathrm{H}-\text { glc- } \mathrm{H}_{2} \mathrm{O}-\text {-cou }\right]^{-}, \\
\text {193.0547, } 191.0361,163.0403, \\
119.0510\end{array}$ & $\begin{array}{l}\text { 6-O-E-p-coumaroyl } \\
\text { scandoside methyl } \\
\text { ester }\end{array}$ & $\begin{array}{l}\text { Herb, } \\
\text { Plasma }\end{array}$ \\
\hline 21 & 12.78 & $\mathrm{C}_{37} \mathrm{H}_{38} \mathrm{O}_{19}$ & $\begin{array}{l}268 \\
328\end{array}$ & $\begin{array}{l}809.18960 \\
(-0.4)\end{array}$ & $\begin{array}{l}787.20863 \\
(+0.8)\end{array}$ & $\begin{array}{l}785.19407 \\
(+0.8)\end{array}$ & $\begin{array}{l}449.1072\left[\mathrm{M}+\mathrm{H}-\text { fer}- \text { glc }^{+},\right. \\
287.0553 \\
\text { [M+H-fer-glc-gall }^{+}, \\
177.0542,145.0275\end{array}$ & $\begin{array}{l}609.1492\left[\mathrm{M}-\mathrm{H}-\mathrm{fer}^{-}\right]^{-}, 429.0849 \\
{\left[\mathrm{M}-\mathrm{H}-\text { fer-glc- } \mathrm{H}_{2} \mathrm{O}^{-}{ }^{-}, 285.0431\right.} \\
{\text { [M-H-fer-glc-gal }]^{-},}, 255.0303 \\
227.0367\end{array}$ & $\begin{array}{l}\text { Kaempferol-3-O-[2-O-(6- } \\
O-E \text {-feruloyl)- } \beta \text {-D- } \\
\text { glucopyranosyl]- } \beta \text {-D- } \\
\text { galactopyranoside }\end{array}$ & $\begin{array}{l}\text { Herb, } \\
\text { Plasma }\end{array}$ \\
\hline 22 & 12.87 & $\mathrm{C}_{27} \mathrm{H}_{32} \mathrm{O}_{14}$ & 299 & $\begin{array}{l}603.16862 \\
(+0.3)\end{array}$ & $\begin{array}{l}581.1864 \\
(-0.2)\end{array}$ & $\begin{array}{l}579.17408 \\
(3.7)\end{array}$ & & $\begin{array}{l}399.1111\left[\mathrm{M}-\mathrm{H}-\mathrm{glc}-\mathrm{H}_{2} \mathrm{O}^{-}{ }^{-},\right. \\
223.0617[\mathrm{M}-\mathrm{H}-\text { fer-glc- } \\
\text { 193.0532, } \\
\text { 119.0350 }\end{array}$ & $\begin{array}{l}\text { 6-O-Z- } p \text {-feruloyl } \\
\text { scandoside methyl } \\
\text { ester }\end{array}$ & Herb \\
\hline 23 & 13.26 & $\mathrm{C}_{27} \mathrm{H}_{32} \mathrm{O}_{14}$ & 299 & $\begin{array}{l}603.16862 \\
(+0.3)\end{array}$ & $\begin{array}{l}581.18627 \\
(-0.4)\end{array}$ & $\begin{array}{l}579.17283 \\
(+1.5)\end{array}$ & & $\begin{array}{l}\left.\text { 399.1083 [M-H-glc- } \mathrm{H}_{2} \mathrm{O}\right]^{-}, \\
223.0622\left[\mathrm{M}-\mathrm{H}-\mathrm{fer}-\mathrm{glc}_{2}-\mathrm{H}_{2} \mathrm{O}^{-},\right. \\
\text {193.0518, } 191.0375,134.0382, \\
119.0348\end{array}$ & $\begin{array}{l}\text { 6-O-E- } p \text {-feruloyl } \\
\text { scandoside methyl } \\
\text { ester }\end{array}$ & Herb \\
\hline 24 & 14.64 & $\mathrm{C}_{15} \mathrm{H}_{10} \mathrm{O}_{7}$ & $\begin{array}{l}254 \\
370\end{array}$ & & $\begin{array}{l}303.04973 \\
(-0.7)\end{array}$ & $\begin{array}{l}301.03565 \\
(+0.9)\end{array}$ & $\begin{array}{l}285.0385\left[\mathrm{M}+\mathrm{H}-\mathrm{H}_{2} \mathrm{O}\right]^{+}, \\
229.0479 \\
{\left[\mathrm{M}+\mathrm{H}-\mathrm{H}_{2} \mathrm{O}-2 \mathrm{CO}^{+}\right.} \\
177.0543,153.0176\end{array}$ & $\begin{array}{l}286.0583\left[\mathrm{M}-\mathrm{H}-\mathrm{CH}_{3}\right]^{-}, \\
179.0001,151.0058\end{array}$ & Quercetin & $\begin{array}{l}\text { Herb, } \\
\text { Plasma, } \\
\text { Kidney }\end{array}$ \\
\hline 25 & 14.78 & $\mathrm{C}_{16} \mathrm{H}_{12} \mathrm{O}_{5}$ & & & $\begin{array}{l}285.07565 \\
(-0.3)\end{array}$ & $\begin{array}{l}283.06143 \\
(+0.8)\end{array}$ & 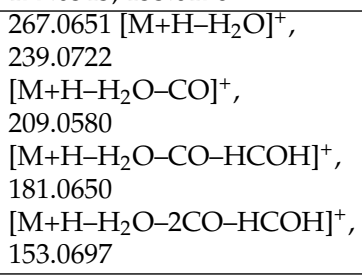 & $\begin{array}{l}268.0399\left[\mathrm{M}-\mathrm{H}-\mathrm{CH}_{3}\right]^{-}, \\
239.0364\left[\mathrm{M}-\mathrm{H}-\mathrm{CO}_{2}\right]^{-}, 211.0432 \\
{\left[\mathrm{M}-\mathrm{H}-\mathrm{CO}_{2}-\mathrm{CO}\right]^{-}, 195.0471} \\
{\left[\mathrm{M}-\mathrm{H}-2 \mathrm{CO}_{2}\right]^{-}}\end{array}$ & $\begin{array}{l}\text { 2,6-Dihydroxy-3-methyl- } \\
\text { 4-methoxyanthraquinone } \\
\text { or isomer }\end{array}$ & $\begin{array}{l}\text { Herb, } \\
\text { Plasma }\end{array}$ \\
\hline 26 & 15.01 & $\mathrm{C}_{15} \mathrm{H}_{10} \mathrm{O}_{6}$ & $\begin{array}{l}260 \\
370\end{array}$ & & 287.0548 & $\begin{array}{l}285.04049 \\
(+0.1)\end{array}$ & $\begin{array}{l}\text { 165.0170, 153.0170, } \\
121.0274\end{array}$ & $\begin{array}{l}\left.\text { 179.0001 [M-H-2 } \mathrm{CO}_{2}\right]^{-}, \\
151.0025,107.0129,121.0284\end{array}$ & Kaempferol & $\begin{array}{l}\text { Herb, } \\
\text { Plasma, } \\
\text { Kidney }\end{array}$ \\
\hline 27 & 15.98 & $\mathrm{C}_{15} \mathrm{H}_{10} \mathrm{O}_{4}$ & & & $\begin{array}{l}255.06504 \\
(-0.6)\end{array}$ & $\begin{array}{l}253.05088 \\
(+1.0)\end{array}$ & $\begin{array}{l}227.0675[\mathrm{M}+\mathrm{H}-\mathrm{CO}]^{+}, \\
209.0570 \\
{\left[\mathrm{M}+\mathrm{H}-\mathrm{CO}-\mathrm{H}_{2} \mathrm{O}\right]^{+},} \\
199.0738[\mathrm{M}+\mathrm{H}-2 \mathrm{CO}]^{+}, \\
171.0792[\mathrm{M}+\mathrm{H}-3 \mathrm{CO}]^{+}, \\
153.0678 \\
{\left[^{+}+\mathrm{H}-3 \mathrm{CO}-\mathrm{H}_{2} \mathrm{O}\right]^{+}}\end{array}$ & $\begin{array}{l}224.0497[\mathrm{M}+\mathrm{H}-\mathrm{CHO}]^{-}, \\
183.0670 \\
{\left[\mathrm{M}+\mathrm{H}-\mathrm{CHO}-\mathrm{CH}_{3} \mathrm{CO}\right]^{-}}\end{array}$ & $\begin{array}{l}\text { 1-Methoxy-2- } \\
\text { hydroxyanthraquinone }\end{array}$ & $\begin{array}{l}\text { Herb, } \\
\text { Plasma, } \\
\text { Kidney }\end{array}$ \\
\hline
\end{tabular}


Table 1. Cont

\begin{tabular}{|c|c|c|c|c|c|c|c|c|c|c|}
\hline No. & Rt (min) & $\begin{array}{l}\text { Molecular } \\
\text { Formula }\end{array}$ & $\begin{array}{l}\lambda \max \\
(\mathrm{nm})\end{array}$ & {$[\mathrm{M}+\mathrm{Na}]^{+}$} & {$[\mathbf{M}+\mathbf{H}]^{+}$} & {$[\mathbf{M}-\mathbf{H}]^{-}$} & $\begin{array}{l}\text { Major Fragmentors in } \\
\text { Positive Mode }\end{array}$ & $\begin{array}{l}\text { Major Fragmentors in } \\
\text { Negative Mode }\end{array}$ & Identification & Source \\
\hline 28 & 16.17 & $\mathrm{C}_{16} \mathrm{H}_{12} \mathrm{O}_{5}$ & & & $\begin{array}{l}285.07548 \\
(-1.0)\end{array}$ & $\begin{array}{l}283.05952 \\
(-5.9)\end{array}$ & $\begin{array}{l}270.0544\left[\mathrm{M}+\mathrm{H}-\mathrm{CH}_{3}\right]^{+}, \\
252.0400 \\
{\left[\mathrm{M}+\mathrm{H}-\mathrm{CH}_{3}-\mathrm{H}_{2} \mathrm{O}\right]^{+},} \\
224.0463 \\
{\left[\mathrm{M}+\mathrm{H}-\mathrm{CH}_{3}-\mathrm{H}_{2} \mathrm{O}-\mathrm{CO}^{+}\right.} \\
196.0500 \\
{\left[\mathrm{M}+\mathrm{H}-\mathrm{CH}_{3}-\mathrm{H}_{2} \mathrm{O}-2 \mathrm{CO}\right]^{+}} \\
168.0568,139.0521\end{array}$ & $\begin{array}{l}268.0376\left[\mathrm{M}-\mathrm{H}-\mathrm{CH}_{3}\right]^{-}, \\
253.0146\left[\mathrm{M}+\mathrm{H}-\mathrm{HCOH}^{-},\right. \\
240.0432\left[\mathrm{M}-\mathrm{H}-\mathrm{CH}_{3}-\mathrm{CO}\right]^{-}, \\
225.0202 \\
{\left[\mathrm{M}+\mathrm{H}-\mathrm{HCOH}-\mathrm{CO}^{-},\right.} \\
212.0451\left[\mathrm{M}-\mathrm{H}-\mathrm{CH}_{3}-2 \mathrm{CO}\right]^{-}, \\
197.0311 \\
{[\mathrm{M}+\mathrm{H}-\mathrm{HCOH}-2 \mathrm{CO}]^{-},} \\
184.0539,169.0316\end{array}$ & $\begin{array}{l}\text { 2,6-Dihydroxy-3-methyl- } \\
\text { 4-methoxyanthraquinone } \\
\text { or isomer }\end{array}$ & $\begin{array}{l}\text { Herb, } \\
\text { Plasma }\end{array}$ \\
\hline 29 & 16.93 & $\mathrm{C}_{16} \mathrm{H}_{12} \mathrm{O}_{4}$ & & & $\begin{array}{l}269.08036 \\
(-1.8)\end{array}$ & $\begin{array}{l}267.06684 \\
(+2.1)\end{array}$ & $\begin{array}{l}254.0562\left[\mathrm{M}+\mathrm{H}-\mathrm{CH}_{3}\right]^{+}, \\
226.0610\left[\mathrm{M}+\mathrm{H}-\mathrm{CH}_{3}-\mathrm{CO}\right]^{+}, \\
197.0582 \\
{\left[\mathrm{M}+\mathrm{H}-\mathrm{CH}_{3}-\mathrm{CO}-\mathrm{CHO}\right]^{+},} \\
181.0636,152.0609\end{array}$ & $\begin{array}{l}252.0534\left[\mathrm{M}+\mathrm{H}-\mathrm{CH}_{3}\right]^{-}, \\
224.0485\left[\mathrm{M}+\mathrm{H}-\mathrm{CH}_{3}-\mathrm{CO}\right]^{-}\end{array}$ & $\begin{array}{l}\text { 1-Methoxy-2-hydroxy-3- } \\
\text { methylanthraquinone }\end{array}$ & $\begin{array}{l}\text { Herb, } \\
\text { Plasma }\end{array}$ \\
\hline 30 & 17.53 & $\mathrm{C}_{16} \mathrm{H}_{12} \mathrm{O}_{5}$ & & & 285.0754 & $\begin{array}{l}283.06143 \\
(+0.8)\end{array}$ & $\begin{array}{l}270.0521\left[\mathrm{M}+\mathrm{H}-\mathrm{CH}_{3}\right]^{+}, \\
242.0551\left[\mathrm{M}+\mathrm{H}-\mathrm{CH}_{3}-\mathrm{CO}\right]^{+}, \\
214.0610\left[\mathrm{M}+\mathrm{H}-\mathrm{CH}_{3}-2 \mathrm{CO}\right]^{+} \\
187.0594,169.0646\end{array}$ & $\begin{array}{l}268.0376\left[\mathrm{M}-\mathrm{H}-\mathrm{CH}_{3}\right]^{-} \\
240.0432\left[\mathrm{M}-\mathrm{H}-\mathrm{CH}_{3}-\mathrm{CO}\right]^{-} \\
212.0451\left[\mathrm{M}-\mathrm{H}-\mathrm{CH}_{3}-2 \mathrm{CO}\right]^{-}\end{array}$ & $\begin{array}{l}\text { 2,6-Dihydroxy-3-methyl- } \\
\text { 4-methoxyanthraquinone } \\
\text { or isomer }\end{array}$ & $\begin{array}{l}\text { Herb, } \\
\text { Plasma }\end{array}$ \\
\hline 31 & 18.21 & $\mathrm{C}_{18} \mathrm{H}_{28} \mathrm{O}_{2}$ & & & $\begin{array}{l}277.21599 \\
(-0.8)\end{array}$ & $\begin{array}{l}275.20167 \\
(+0.1)\end{array}$ & $\begin{array}{l}\text { 259.2041, 235.1693, 171.1151, } \\
\text { 149.1311, 135.1159, } 121.1088, \\
\text { 107.0859, 93.0709, } 79.0561\end{array}$ & $231.2161\left[\mathrm{M}-\mathrm{H}-\mathrm{CO}_{2}\right]^{-}$ & Unidentified & $\begin{array}{l}\text { Herb, } \\
\text { Plasma, } \\
\text { Kidney }\end{array}$ \\
\hline 32 & 18.56 & $\mathrm{C}_{18} \mathrm{H}_{30} \mathrm{O}_{2}$ & & & $\begin{array}{l}297.23200 \\
(+0.5)\end{array}$ & $\begin{array}{l}277.21874 \\
(+5.2)\end{array}$ & $\begin{array}{l}\text { 261.2217, 243.2109, 223.1691, } \\
\text { 187.1478, 173.1321, 151.1482, } \\
\text { 137.1328, 123.1172, 109.1021, } \\
95.0807,81.0720\end{array}$ & & Unidentified & $\begin{array}{l}\text { Herb, } \\
\text { Plasma, } \\
\text { Kidney }\end{array}$ \\
\hline 33 & 19.07 & $\mathrm{C}_{19} \mathrm{H}_{26} \mathrm{O}_{5}$ & & $\begin{array}{l}357.16079 \\
(-0.4)\end{array}$ & $\begin{array}{l}335.18563 \\
(+1.0)\end{array}$ & & $\begin{array}{l}\text { 163.0751, 145.0643, 115.0543, } \\
91.0555,71.0515\end{array}$ & & Unidentified & Herb \\
\hline 34 & 20.13 & $\mathrm{C}_{18} \mathrm{H}_{30} \mathrm{O}_{2}$ & & & $\begin{array}{l}279.23202 \\
(+0.6)\end{array}$ & $\begin{array}{l}277.21874 \\
(+5.2)\end{array}$ & $\begin{array}{l}\text { 261.2214, 243.2105, 223.1696, } \\
\text { 187.1479, 173.1322, 151.1470, } \\
\text { 137.1321, 123.1173, 109.1020, } \\
95.0867,81.0716,67.0572\end{array}$ & $\begin{array}{l}259.2064\left[\mathrm{M}-\mathrm{H}-\mathrm{H}_{2} \mathrm{O}\right]^{-} \\
233.2282\left[\mathrm{M}-\mathrm{H}-\mathrm{CO}_{2}\right]^{-}\end{array}$ & Unidentified & $\begin{array}{l}\text { Herb, } \\
\text { Plasma, } \\
\text { Kidney }\end{array}$ \\
\hline 35 & 20.68 & $\mathrm{C}_{30} \mathrm{H}_{48} \mathrm{O}_{3}$ & & & $\begin{array}{l}457.36734 \\
(-0.6)\end{array}$ & $\begin{array}{l}455.35279 \\
(-0.6)\end{array}$ & $\begin{array}{l}\text { 439.3564, 411.3615, 393.3508, } \\
341.2846,315.2694,297.2575, \\
\text { 249.1844, 231.2106, 203.1791, } \\
\text { 163.1476, 149.1316, 121.1009, } \\
95.0863,81.0713\end{array}$ & $407.3300[\mathrm{M}-\mathrm{H}-\mathrm{HCOOH}]^{-}$ & Ursolic acid & Herb \\
\hline
\end{tabular}


As shown in Figure 3, anthraquinone compounds did not showed obviously UV absorption in DAD spectra, but with good mass response in positive ion mode. They were always characterized by losses of $\mathrm{CH}_{3}(15 \mathrm{Da}) \mathrm{CO}(28 \mathrm{Da}), \mathrm{H}_{2} \mathrm{O}(18 \mathrm{Da})$, and $\mathrm{HCOH}(30 \mathrm{Da})$. Compounds 25,28 and 30 gave the same protonated molecular ion at $\mathrm{m} / \mathrm{z} 285$ in positive ion mode and $\mathrm{m} / \mathrm{z} 283$ in negative ion mode, with molecular formula of $\mathrm{C}_{16} \mathrm{H}_{12} \mathrm{O}_{5}$. The losses of neutral ions of $18 \mathrm{Da}, 28$ $\mathrm{Da}$ and $30 \mathrm{Da}$ in MS spectra showed anthraquinone compounds characteristics. Thus they were identified as 2,6-Dihydroxy-3-methyl-4-methoxyanthraquinone or its isomer referring to previous report [16], and were necessary to be confirmed by NMR. Compound 27 gave protonated ion at $m / z 255.06504\left(\mathrm{C}_{15} \mathrm{H}_{10} \mathrm{O}_{4}\right)$, and 30 Da less than compound 25, suggesting the lack of methoxy group. Compound 29 showed molecular formula of $\mathrm{C}_{16} \mathrm{H}_{12} \mathrm{O}_{4}$ with the protonated ion at 269.08036, and 14 Da more than compound 27 suggested the presence of methyl group. In according with the report [17,18], compounds 27 and 29 were identified as 1-methoxy-2-hydroxyanthraquinone and 1-methoxy-2-hydroxy-3-methylanthraquinone, respectively.

\subsection{Discussion}

LPS as an important inflammatory factor, could induce endotoxemia, shock and multiple organ dysfunction syndromes. During LPS-induced inflammatory process, multiple endogenous inflammatory mediators were produced in response to LPS, and the immune responses and organic functions were modulated [19-21]. The inflammatory model induced by LPS was commonly used to investigate the effect of herbal medicines and its bioactive constituents on renal inflammation [21-24]. In various acute and chronic renal inflammations, renal tubular epithelial cells actively participate in the process of glomerular sclerosis and renal fibrosis via producing all kinds of inflammatory cytokines, chemokines and extracellular matrix [25]. It has been reported that macrophages are involved in the productions of pro-inflammatory cytokines [26]. Macrophage infiltration is considered to be a hallmark of all kinds of injury, and macrophage infiltration into the kidney is found as one of the initial events of renal disease [27]. In this study, the results of histological and immunohistochemical examination of renal section clearly showed the evidence that $H$. diffusa extract had protective effect against renal inflammation induced by LPS, at the dose-dependent manner.

TNF- $\alpha$, as the pro-inflammatory cytokine, activates other cytokines and therefore plays an important role in the pathogenesis of LPS-induced renal damage. A way to protect against LPS-induced inflammation is to inhibit TNF- $\alpha$ action [28,29]. Similarly, IL-1 $\beta$ could also stimulate the production of other pro-inflammatory cytokines, such as IL-6 [30,31]. It is thought that increasing production of IL-1 $\beta$ in renal tissues indicates the pathological development of inflammation, while increasing production of IL-6 is involved in the chronic processes and can suppress the synthesis of IL-1 $\beta$ in the second phase of the inflammatory response [32]. H. diffusa could down-regulate the levels of pro-inflammatory cytokines in serum and renal tissue, therefore markedly ameliorating the local immune response induced by LPS. Moreover, TNF- $\alpha$ stimulates the production of chemokines, such as MCP-1. MCP-1 is chemotactic for multiple leukocytes, including neutrophils, monocytes and natural killer cells [33,34]. The anti-inflammatory cytokine IL-10, which suppresses the production of pro-inflammatory cytokines and chemokines mainly through inhibition of innate immune cell activation, was up-regulated and therefore ameliorated LPS-induced renal damage in animal models [35]. The inflammatory reaction was evaluated by the production levels of pro-inflammatory cytokines, anti-inflammatory cytokine and chemokine in serum and renal tissues. The results demonstrated that water extract of $H$. diffusa could dose-dependently attenuate the levels of TNF- $\alpha$, IL-1 $\beta$, IL-6, MCP-1, and IL-10, which are associated with the improvement of function in nephropathy tissues.

A series of studies showed that flavonoids, iridoids and anthraquinones from herbal medicine have anti-inflammatory effect [36-41]. Kaempferol could suppress the activation of inflammatory nuclear factor- $\mathrm{KB}(\mathrm{NF}-\mathrm{kB})$ transcription factor through nuclear factor-inducing kinase/I $\kappa B$ kinase and mitogen-activated protein kinase (MAPK) in aged rat kidney [42]. Quercetin had the ability 
to attenuate activation of NF- $\mathrm{KB}$ and inhibited IL-1-triggered MCP-1 expression via suppression of NF- $\mathrm{BB}$ [43]. Rutin, a quercetin glycoside, attenuated renal inflammation and apoptosis by reducing NF- $\mathrm{kB}, \mathrm{TNF}-\alpha$ and caspase-3 expression [44]. The 5-glucoside of quercetin named saxifragin exerted anti-inflammatory activity by inhibiting NF- $\mathrm{kB}$, caspase-1, and MAPK activation [45]. Emodin, an anthraquinone derivative could activate peroxisome proliferator-activated receptor $\gamma$ (PPAR- $\gamma$ ), and inhibit TLR2-mediated NF-KB signal pathway, thereby contributing to the immune inflammation regulation of emodin in LPS-induced acute kidney injury [46,47]. Iridoid glycosides possessed anti-inflammatory and immunomodulatory effects by suppressing transmembrane signal transmission in NF- $\mathrm{K}$ Bp65 pathway, decreasing the expressions of MCP-1 and $\alpha$-smooth muscle actin $(\alpha-\mathrm{SMA})$ to modulate the productions of pro-inflammatory mediators in nephropathy tissue [48-50]. The studies showed that inhibition of inflammatory response and induction of apoptotic signal via natural products could be useful approaches for prevention and cure of LPS-induced renal damage. In this study, the chemical profiles indicated that water extract of $H$. diffusa mainly contained these three chemotypes by UPLC-DAD-Q-TOF-MS/MS method. As high doses of extract of H. diffusa obviously protected renal tissues, suppressed the productions of the anti-inflammatory cytokines and chemokine, and promoted the production of anti-inflammatory cytokine, chemical profiles of the absorbed constituents of $H$. diffusa were necessary to elucidate the effective compounds in vivo. In the study, there were ten iridois, eleven flavonoids and five anthraquinones found in serum from the $H$. diffusa extract treatment groups. Moreover, the flavonoids, including kaempferol, quercetin, three kaempferol glycosides and three quercetin glycosides, were the most chemotype found in nephropathy tissues, indicating that flavonoids might target on the nephropathy tissues and greatly contribute to the suppression of inflammation process. Four iridois and one anthraquinone found in renal tissues suggested that these constituents might also have anti-inflammatory effect. The results of chemical study indicated that flavonoids, iridoids and anthraquinones might be responsible for the anti-inflammatory effect of $H$. diffusa and therefore could ameliorate the renal function and regulate the levels of the cytokines and chemokines through anti-inflammatory responses. However, the quantification of potential bioactive constituents and the method validation are not performed due to lacks of two-third of commercial standards, including three iridois, nine flavonoids, five anthraquinones and three unidentified compounds. Isolation and structural identification of non-commercial potential bioactive constituents from $H$. diffusa are undergoing in order to exactly identify and completely quantify all potential bioactive constituents in serum and renal tissues. In addition, the molecular pathway and anti-inflammatory mechanism of $H$. diffusa extract are still unclear, and thus further study is required according to the previous reports and these results.

\section{Experimental Section}

\subsection{Plant Material}

The materials of $H$. diffusa were collected from Guangzhou Qingping Herbal Market, Guangzhou, China. The materials were authenticated by the authors and dried at room temperature. The materials were stored in Guangzhou Institute of Advanced Technology, Chinese Academy of Sciences, Guangzhou, China.

\subsection{Chemicals and Reagents}

Deacetylasperulosidec acid, scandoside, geniposidic acid, scandoside methyl ester, asperulosidic acid, scopolin and asperuloside were purchased from Chengdu Biopurify Technology Development Co., Ltd. (Chengdu, China). p-courmaric acid, ferulic acid, rutin, quercetin, kaempferol, ursolic acid were from National Institutes for food and drug control (Beijing, China). HPLC-grade methanol was purchased from Fisher Scientific (Pittsburgh, PA, USA). Distilled water was purified by a Milli-Q system (Millipore, Milford, MA, USA). Other reagents were analytical grade. 


\subsection{Sample Preparation}

Thirteen standards for the identification were prepared in HPLC-grade methanol to obtain the standard solutions of deacetylasperulosidec acid $(0.17 \mu \mathrm{g} / \mathrm{mL})$, scandoside $(0.21 \mu \mathrm{g} / \mathrm{mL})$, geniposidic acid $(0.20 \mu \mathrm{g} / \mathrm{mL})$, scandoside methyl ester $(0.19 \mu \mathrm{g} / \mathrm{mL})$, asperulosidic acid $(0.20 \mu \mathrm{g} / \mathrm{mL})$, scopolin $(0.18 \mu \mathrm{g} / \mathrm{mL})$, asperuloside $(0.20 \mu \mathrm{g} / \mathrm{mL}), p$-courmaric acid $(0.20 \mu \mathrm{g} / \mathrm{mL})$, ferulic acid $(0.19 \mu \mathrm{g} / \mathrm{mL})$, rutin $(0.21 \mu \mathrm{g} / \mathrm{mL})$, quercetin $(0.21 \mu \mathrm{g} / \mathrm{mL})$, kaempferol $(0.20 \mu \mathrm{g} / \mathrm{mL})$, and ursolic acid $(0.19 \mu \mathrm{g} / \mathrm{mL})$, respectively. All standard solutions were stored at $4{ }^{\circ} \mathrm{C}$ until used.

The dried $H$. diffusa were cut into $1 \mathrm{~cm}$ pieces, weighed $(100 \mathrm{~g})$ and boiled in $1 \mathrm{~L}$ water for $1 \mathrm{~h}$; after filtration, the extraction was repeated twice; the combined filtrates were evaporated in vacuum at $70{ }^{\circ} \mathrm{C}$ (EYELA N-1001, Tokyo, Japan). The concentrated solution $(100 \mathrm{~mL})$ was added to ethanol $\left(300 \mathrm{~mL}\right.$ ) and stored at $4{ }^{\circ} \mathrm{C}$ overnight; after filtration, ethanol in the solution was evaporated at $50{ }^{\circ} \mathrm{C}$; and, finally, $100 \mathrm{~mL}$ of a brown, concentrated extract (equal to $1.0 \mathrm{~g}$ raw material $/ \mathrm{mL}$ ) were obtained and stored at $4{ }^{\circ} \mathrm{C}$ until used.

\subsection{Animals and Experiment Design}

Kunming mice in the body weights of 18-20 g were purchased from the Experimental Animal Center of Southern Medical University (Guangzhou, China). Mice were fed on standard laboratory diet and water at libitum, and kept in $12 \mathrm{~h}$ dark/light cycle room at $21 \pm 5^{\circ} \mathrm{C}$ with a relative humidity of $55 \% \pm 10 \%$ for one week before used. All experimental protocols were approved by the Animal Care and Use Committee of Southern Medical University (No. 2014A03031028, Guangzhou, China).

Mice were randomly divided into 5 groups $(n=8)$. In a week, the control group and the model group was injected intraperitoneally with sterile physiological saline $(0.2 \mathrm{~mL} / 20 \mathrm{~g}$ bw, i.p. (intraperitoneal injection)); the treatment groups received the water extract of $H$. diffusa (equal to $1.25,2.5$ or $5.0 \mathrm{~g} / \mathrm{kg}$ bw, i.p.). Except for the control group, the other four groups were injected intraperitoneally with LPS ( $3 \mathrm{mg} / \mathrm{kg} \mathrm{bw})$ after the sample injection on the fifth day. LPS injection was continued for three consecutive days. On the seventh day, all mice from each group were anesthetized by ether after LPS injection. Blood samples were taken from abdominal aorta. The serum was obtained by centrifugation at $13,000 \times \mathrm{g}$ at $4{ }^{\circ} \mathrm{C}$ for $10 \mathrm{~min}$ and stored at $-80{ }^{\circ} \mathrm{C}$ until analysis. After kidneys were removed carefully, one kidney was fixed with $10 \%$ formaldehyde for histological and immunohistochemical examinations, and the other one was frozen in liquid nitrogen for subsequent analysis. The frozen kidney was homogenized in ice-cold Tris- $\mathrm{HCl}$ buffer $(\mathrm{pH}=7.4)$ containing protease inhibitors. The homogenized samples were centrifuged at $13,000 \times g$ at $4{ }^{\circ} \mathrm{C}$ for $20 \mathrm{~min}$. The supernatants were collected and frozen at $-80^{\circ} \mathrm{C}$ until assay.

\subsection{Preparation of Bioanalytical Samples}

An aliquot of $160 \mu \mathrm{L}$ of combined plasma ( $20 \mu \mathrm{L}$ per mouse) was mixed with $600 \mu \mathrm{L}$ acetonitrile and vortexed for $1 \mathrm{~min}$. After centrifuging at $13,000 \times \mathrm{g}$ at $4{ }^{\circ} \mathrm{C}$ for $30 \mathrm{~min}$, the supernatant was transferred into a fresh tube and evaporated with nitrogen. The residue dissolved in a $160 \mu \mathrm{L}$ mobile phase was vortexed for $3 \mathrm{~min}$ and centrifuged at $13,000 \times \mathrm{g}$ for $30 \mathrm{~min}$. Finally, an aliquot of $10 \mu \mathrm{L}$ of the supernatant were injected into the UFLC/DAD/Q-TOF-MS/MS system. An aliquot of $160 \mu \mathrm{L}$ of mixed kidney supernatants ( $20 \mu \mathrm{L}$ per mouse) was prepared as same method as the combined plasma.

\subsection{Bioanalytical Method}

UFLC analysis was performed on a Shimadzu UFLC XR instrument (Shimadzu Corp., Kyoto, Japan), consisting of a binary pump, an autosampler, a column oven and a diode-array detector. Samples were separated on a Phenomenex Kinetex column $(2.1 \mathrm{~mm}$ i.d. (internal diameter) $\times 100 \mathrm{~mm}$, $2.6 \mu \mathrm{m}$, Phenomenex, CA, USA). The mobile phase consisted of water (A) and methanol (B) both containing $0.1 \%$ formic acid $(v / v)$ using a gradient elution program of $13 \% \mathrm{~B}(0-2 \mathrm{~min}), 13 \%-15 \% \mathrm{~B}$ (2-8 $\mathrm{min}), 15 \%-23 \% \mathrm{~B}(8-12 \mathrm{~min}), 23 \%-98 \% \mathrm{~B}(12-20 \mathrm{~min})$ and $98 \% \mathrm{~B}$ (20-25 min). A 2-min post-run 
time was set to equilibrate the column. The flow rate was kept at $0.3 \mathrm{~mL} / \mathrm{min}$. The injected volume was $2 \mu \mathrm{L}$ and the column temperature was set at $40^{\circ} \mathrm{C}$. The DAD detector scanned from 190 to $400 \mathrm{~nm}$. Mass spectrometry was performed on the Triple TOF ${ }^{\mathrm{TM}} 5600$ plus (AB SCIEX, Foster City, CA, USA) a hybrid triple quadrupole time-of-flight mass spectrometer equipped with ESI source. The system was operated with Analyst ${ }^{\circledR}$ TF 1.6 software (AB SCIEX, Foster City, CA, USA). The conditions of MS/MS detector were as follows: ion source gas 155 psi; ion source gas 255 psi; curtain gas 30 psi; Source temperature $550^{\circ} \mathrm{C}$; ion spray voltage floating $4500 \mathrm{~V}$; collision energy $35 \mathrm{~V}$; collision energy spread $15 \mathrm{~V}$; declustering potential $80 \mathrm{~V}$. TOF-MS range was set at $m / z 100-1000$ and product ions mass range was set at $m / z 50-1000$. Both positive and negative ion modes were used for compounds ionization. Nitrogen was used as nebulizer and auxiliary gas.

The UFLC-DAD-Q-TOF-MS/MS data of samples were extracted and analyzed by PeakView software (AB SCIEX, Foster City, CA, USA), mainly with the XIC manager tool that provided the quasi-molecular weight, mass errors and isotope pattern fit. The predicted formula with errors less than $\pm 5 \mathrm{ppm}$ was applied to compare with that of the compounds reported in Rubiaceae. The tentative identification of each compound was further guided by MS/MS spectrum (AB SCIEX, Foster City, CA, USA) that could determine the possible elemental compositions of the fragment ions and propose the fragmentation pathways.

\subsection{Histological Examination}

Kidneys were fixed with $10 \%$ formalin for $24 \mathrm{~h}$ in a shaker and embedded in paraffin. The paraffin blocks were cut into $4-\mu \mathrm{m}$-thick sections using a microtome and then stained with periodic acid-Schiff (PAS) reagents for histological examination. Renal sections were evaluated at $400 \times$ magnification.

\subsection{Immunohistochemical Examination}

After embedded renal tissues were deparaffinised in xylene and rehydrated, and the immunohistochemical examination was performed according to the manufacturer's instruction (GTVisionTMI Detection System, Gene Tech, Shanghai, China). Eight random fields were chosen and evaluated at $200 \times$ magnifications.

\subsection{Determination of Cytokines}

Concentrations of TNF- $\alpha$, IL-1 $\beta$, IL-6, IL-10 and MCP-1 in serum and renal tissues were determined using mouse-specific enzyme-linked immunosorbent assay (ELISA) kits (NeoBioscience, Shenzhen, China). Each analysis was performed according to the manufacturer's instruction, and the concentrations of cytokines were calculated according to the standard curves.

\subsection{Statistical Analysis}

Results were shown as mean \pm standard deviation (SD) for each experiment. The data obtained were analyzed using one-way analysis of variance (ANOVA) followed by Tukey's multiple comparison test (SPSS version 19.0, IBM, New York, NY, USA). A $p$ value $<0.05$ was considered as significant difference.

\section{Conclusions}

In this study, the results showed that water extract of $H$. diffusa could protect against the renal damage induced by LPS through down-regulating the levels of TNF- $\alpha$, IL- $1 \beta$, IL-6, and MCP-1, as well as up-regulating the level of anti-inflammatory cytokine IL-10. It also demonstrated that the main constituents, flavonoids, iridoids and anthraquinones, were possibly responsible for the anti-inflammatory effect of $H$. diffusa. 
Acknowledgments: This work was supported by Guangdong Natural Science Foundation (2014A03031028) and Foshan Medical Scientific Research Foundation (201108048). The UFLC-DAD-Q-TOF-MS/MS used in this work was provided by Guangzhou Quality R\&D center of Traditional Chinese Medicine, School of Life Sciences, Sun Yat-Sen University, Guangzhou 510275, PR China.

Author Contributions: Jian-Hong Ye, Jing-Yu He and Meng-Hua Liu designed the research; Jian-Hong Ye, Jing-Yu He, Xu-Lin Zhang and Meng-Hua Liu performed the experiments; Jing-Yu He and Meng-Hua Liu analyzed the experimental data and wrote the manuscript.

Conflicts of Interest: The authors declare no conflict of interest.

\section{References}

1. Editorial Board of Flora of China. The Chinese Academy of Sciences. In Flora of China; Science Press: Beijing, China, 1999; Volume 71, p. 75.

2. Nanjing University of Chinese Medicine. Dictionary of Chinese Traditional Medicine (Zhong. Yao Da Ci Dian), 2nd ed.; Shanghai Scientific \& Technical Publishers: Shanghai, China, 2006; pp. 1039-1041.

3. Xu, G.H.; Kim, Y.H.; Chi, S.W.; Choo, S.J.; Ryoo, I.J.; Ahn, J.S.; Yoo, I.D. Evaluation of human neutrophil elastase inhibitory effect of iridoid glycosides from Hedyotis diffusa. Bioorg. Med. Chem. Lett. 2010, 20, 513-515. [CrossRef] [PubMed]

4. Lin, C.C.; Ng, L.T.; Yang, J.J.; Hsu, Y.F. Anti-inflammatory and hepatoprotective activity of Peh-Hue-Juwa-Chi-Cao in male rats. Am. J. Chin. Med. 2002, 30, 225-234. [CrossRef] [PubMed]

5. Wang, N.; Li, D.Y.; Niu, H.Y.; Zhang, Y.; He, P.; Wang, J.H. 2-Hydroxy-3-methylanthraquinone from Hedyotis diffusa Willd induces apoptosis in human leukemic U937 cells through modulation of MAPK pathways. Arch. Pharm. Res. 2013, 36, 752-758. [CrossRef] [PubMed]

6. Liu, Z.; Liu, M.; Liu, M.; Li, J.C. Methylanthraquinone from Hedyotis diffusa WILLD induces Ca ${ }^{2+}$-mediated apoptosis in human breast cancer cells. Toxicol. Vitro 2010, 24, 142-147. [CrossRef] [PubMed]

7. Niu, Y.; Meng, Q.X. Chemical and preclinical studies on Hedyotis diffusa with anticancer potential. J. Asian Nat. Prod. Res. 2013, 15, 550-565. [CrossRef] [PubMed]

8. Liu, E.H.; Zhou, T.; Li, G.B.; Li, J.; Huang, X.N.; Pan, F.; Gao, N. Characterization and identification of iridoid glucosides, flavonoids and anthraquinones in Hedyotis diffusa by high-performance liquid chromatography/electrospray ionization tandem mass spectrometry. J. Sep. Sci. 2012, 35, $263-272$. [CrossRef] [PubMed]

9. Li, C.M.; Zhao, Y.Y.; Guo, Z.M.; Zhang, X.L.; Xue, X.Y.; Liang, X.M. Effective 2D-RPLC/RPLC enrichment and separation of micro-components from Hedyotis diffusa Willd and characterization by using ultra-performance liquid chromatography/quadrupole time-of-flight mass spectrometry. J. Pharm. Biomed. Anal. 2014, 99, 35-44. [CrossRef] [PubMed]

10. Ferreres, F.; Gil-Izquierdo, A.; Andrade, P.B.; Valentão, P.; Tomás-Barberán, F.A. Characterization of C-glycosyl flavones $\mathrm{O}$-glycosylated by liquid chromatography-tandem mass spectrometry. J. Chromatogr. A 2007, 1161, 214-223. [CrossRef] [PubMed]

11. Truchado, P.; Vit, P.; Ferreres, F.; Tomas-Barberan, F. Liquid chromatography-tandem mass spectrometry analysis allows the simultaneous characterization of C-glycosyl and $O$-glycosyl flavonoids in stingless bee honeys. J. Chromatogr. A 2011, 1218, 7601-7607. [CrossRef] [PubMed]

12. Kim, Y.; Park, E.J.; Kim, J.; Kim, Y.; Kim, S.R.; Kim, Y.Y. Neuroprotective constituents from Hedyotis diffusa. J. Nat. Prod. 2001, 64, 75-78. [CrossRef] [PubMed]

13. Lu, C.M.; Yang, J.J.; Wang, P.Y.; Lin, C.C. A new acylated flavonol glycoside and antioxidant effects of Hedyotis diffusa. Planta Med. 2000, 66, 374-377. [CrossRef] [PubMed]

14. Zhang, H.J.; Chen, Y.G.; Huang, R. Study on flavonoids from Hedyotis diffusa willia. J. Chin. Med. Mater. 2005, 28, 385-387.

15. Hamzah, A.S.; Aimi, N.; Lajis, N.H.J. Constituents of Hedyotis herbacea (Rubiaceae). Biochem. Syst. Ecol. 1996, 24, 273. [CrossRef]

16. Permana, D.; Lajis, N.H.; Othman, A.G.; Ali, A.M.; Aimi, N.; Kitajima, M.; Takayama, H. Anthraquinones from hedyotis herbacea. J. Nat. Prod. 1999, 62, 1430-1431. [CrossRef] [PubMed]

17. Xu, B.J.; Sung, C.K. Chemical constituents and pharmacological activities of Hedyotis diffusa. Nat. Prod. Sci. 2005, 11, 1-9. 
18. Shi, Y.; Wang, C.H.; Gong, X.G. Apoptosis-inducing effects of two anthraquinones from Hedyotis diffusa WILLD. Biol. Pharm. Bull. 2008, 31, 1075-1078. [CrossRef] [PubMed]

19. Bascands, J.L.; Bachvarova, M.; Neau, E.; Schanstra, J.P.; Bachvarov, D. Molecular determinants of LPS-induced acute renal inflammation: Implication of the kinin $\mathrm{B}_{1}$ receptor. Biochem. Biophys. Res. Commun. 2009, 386, 407-412. [CrossRef] [PubMed]

20. Lee, S.; Kim, S.; Kang, K.P.; Moon, S.O.; Sung, M.J.; Kim, D.H.; Kim, H.J.; Park, S.K. Agonist of peroxisome proliferator-activated receptor- $\gamma$, rosiglitazone, reduces renal injury and dysfunction in a murine sepsis model. Nephrol. Dial. Transplant. 2005, 20, 1057-1065. [CrossRef] [PubMed]

21. Hsiang, C.Y.; Cheng, H.M.; Lo, H.Y.; Li, C.C.; Chou, P.C.; Lee, Y.C.; Ho, T.Y. Ginger and zingerone ameliorate lipopolysaccharide-induced acute systemic inflammation in mice, assessed by nuclear factor- $\mathrm{\kappa B}$ bioluminescent imaging. J. Agric. Food Chem. 2015, 63, 6051-6058. [CrossRef] [PubMed]

22. Granholm, N.A.; Cavallo, T. Long-lasting effects of bacterial lipopolysaccharide promote progression of lupus nephritis in NZB/W mice. Lupus 1994, 3, 507-514. [CrossRef] [PubMed]

23. Gao, L.N.; Cui, Y.L.; Wang, Q.S.; Wang, S.X. Amelioration of Danhong iniection on the lipopolysaccharide-stimulated systemic acute inflammatory reaction via multi-target strategy. J. Ethnopharmacol. 2013, 149, 772-782. [CrossRef] [PubMed]

24. Borges, F.R.M.; Silva, M.D.; Cordova, M.M.; Schambach, T.R.; Pizzolatti, M.G.; Santos, A.R.S. Anti-inflammatory action of hydroalcoholic extract, dichloromethane fraction and steroid $\alpha$-spinasterol from Polygala sabulosa in LPS-induced peritonitis in mice. J. Ethnopharmacol. 2014, 151, 144-150. [CrossRef] [PubMed]

25. Segerer, S.; Nelson, P.J.; Schlondorff, D. Chemokines, chemokine receptors, and renal disease: From basic science to pathophysiologic and therapeutic studies. J. Am. Soc. Nephrol. 2000, 11, 152-176. [PubMed]

26. Abraham, A.P.; Ma, F.Y.; Mulley, W.R.; Ozols, E.; Nikolic-Paterson, D.J. Macrophage infiltration and renal damage are independent of matrix metalloproteinase 12 in the obstructed kidney. Nephrology 2012, 17, 322-329. [CrossRef] [PubMed]

27. Liu, C.; Cheng, Z.A.; Wang, Y.M.; Dai, X.Q.; Zhang, J.; Xue, D.Y. Paeoniflorin exerts a nephroprotective effect on concanavalin A-induced damage through inhibition of macrophage infiltration. Diagn. Pathol. 2015, 10, 120-129. [CrossRef] [PubMed]

28. Venkatesha, S.H.; Dudics, S.; Acharya, B.; Moudgil, K.D. Cytokine-Modulating strategies and newer cytokine targets for arthritis therapy. Int. J. Mol. Sci. 2015, 16, 887-906. [CrossRef] [PubMed]

29. Cunningham, P.N.; Dyanov, H.M.; Park, P.; Wang, J.; Newell, K.A.; Quigg, R.J. Acute renal failure in endotoxemia is caused by TNF acting directly on TNF receptor-1 in kidney. J. Immunol. 2002, 168, 5817-5823. [CrossRef] [PubMed]

30. Ramesh, G.; Reeves, W.B. TNF- $\alpha$ mediates chemokine and cytokine expression and renal injury in cisplatin nephrotoxicity. J. Clin. Investig. 2002, 110, 835-842. [CrossRef] [PubMed]

31. Yoshimura, A.; Hanada, T. Regulation of cytokine signaling and inflammation. Cytokine Growth Factor Rev. 2002, 13, 413-421.

32. Patel, N.S.; Chatterjee, P.K.; Di Paola, R.; Mazzon, E.; de Sarro, A.; Cuzzpcrea, S.; Thiemermann, C. Endogenous interleukin-6 enhances the renal injury, dysfunction, and inflammation caused by ischemia/reperfusion. J. Pharmacol. Exp. Ther. 2005, 312, 1170-1178. [CrossRef] [PubMed]

33. Rovin, B.H.; Phan, L.T. Chemotactic factors and renal inflammation. Am. J. Kidney Dis. 1998, 31, 1065-1084. [CrossRef] [PubMed]

34. Banas, B.; Luckow, B.; Moller, M.; Klier, C.; Nelson, P.J.; Schadde, E.; Brigl, M.; Halevy, D.; Holthofer, H.; Reinhart, B.; et al. Chemokine and chemokine receptor expression in a novel human mesangial cell line. J. Am. Soc. Nephrol. 1999, 10, 2314-2322. [PubMed]

35. Moore, K.W.; De Waal Malefyt, R.; Coffman, R.L.; O'Garra, A. Interleukin-10 and the interleukin-10 receptor. Annu. Rev. Immunol. 2001, 19, 683-765. [CrossRef] [PubMed]

36. Matsuzaki, K.; Ishii, R.; Kobiyama, K.; Kitanaka, S. New benzophenone and quercetin galloyl glycosides from Psidium guajava L. J. Nat. Med. 2010, 64, 252-256. [CrossRef] [PubMed]

37. Flamini, R.; Mattivi, F.; de Rosso, M.; Arapitsas, P.; Bavaresco, L. Advanced knowledge of three important classes of grape phenolics: Anthocyanins, stilbenes and flavonols. Int. J. Mol. Sci. 2013, 14, 19651-19669. [CrossRef] [PubMed] 
38. Xiao, W.Q.; Yin, G.J.; Fan, Y.T.; Qiu, L.; Cang, X.F.; Yu, G.; Hu, Y.L.; Xing, M.; Wu, D.Q.; Wang, X.P.; et al. Catalpol ameliorates sodium taurocholate-induced acute pancreatitis in rats via inhibiting activation of nuclear factor кB. Int. J. Mol. Sci. 2014, 15, 11957-11972. [CrossRef] [PubMed]

39. Xiao, M.; Zhu, T.; Zhang, W.; Wang, T.; Shen, Y.C.; Wan, Q.F.; Wen, F.Q. Emodin ameliorates LPS-induced acute lung injury, involving the inactivation of NF-kB in mice. Int. J. Mol. Sci. 2014, 15, 19355-19368. [CrossRef] [PubMed]

40. He, Y.M.; Zhu, S.; Ge, Y.W.; Kazuma, K.; Zou, K.; Cai, S.Q.; Komatsu, K. The anti-inflammatory secoiridoid glycosides from Gentianae Scabrae Radix: The root and rhizome of Gentiana. scabra. J. Nat. Med. 2015, 69, 303-312. [CrossRef] [PubMed]

41. He, Y.M.; Zhu, S.; Ge, Y.W.; Cai, S.Q.; Komatsu, K. Secoiridoid glycosides from the root of Gentiana crassicaulis with inhibitory effects against LPS-induced NO and IL-6 production in RAW264 macrophages. J. Nat. Med. 2015, 69, 366-374. [CrossRef] [PubMed]

42. Park, M.J.; Lee, E.K.; Heo, H.S.; Sung, B.; Kim, M.K.; Lee, J.; Kim, N.D.; Anton, S.; Choi, J.S.; Yu, B.P.; et al. The anti-inflammatory effect of kaempferol in aged kidney tissues: The involvement of nuclear factor- $\mathrm{kB}$ via nuclear factor-inducing kinase/IкB kinase and mitogen-activated protein kinase pathways. J. Med. Food 2009, 12, 351-358. [CrossRef] [PubMed]

43. Ishikawa, Y.; Sugiyama, H.; Stylianou, E.; Kitamura, M. Bioflavonoid quercetin inhibits interleukin-1-induced transcriptional expression of monocyte chemoattractant protein-1 in glomerular cells via suppression of nuclear factor-кB. J. Am. Soc. Nephorl. 1999, 10, 2290-2296.

44. Arjumand, W.; Seth, A.; Sultana, S. Rutin attenuates cisplatin induced renal inflammation and apoptosis by reducing NF-кB, TNF- $\alpha$ and caspase-3 expression in wistar rats. Food Chem. Toxicol. 2011, 49, $2013-2021$. [CrossRef] [PubMed]

45. Cheon, S.Y.; Chung, K.S.; Jeon, E.; Nugroho, A.; Park, H.J.; An, H.J. Anti-inflammatory activity of saxifragin via inhibition of NF-KB involves caspase-1 activation. J. Nat. Prod. 2015, 78, 1579-1585. [CrossRef] [PubMed]

46. Yang, Z.T.; Zhou, E.S.; Wei, D.; Li, D.P.; Wei, Z.K.; Zhang, W.; Zhang, X.C. Emodin inhibits LPS-induced inflammatory response by activating PPAR- $\gamma$ in mouse mammary epithelial cells. Int. Immunopharmacol. 2014, 21, 354-360. [CrossRef] [PubMed]

47. Li, Y.; Xiong, W.J.; Yang, J.; Zhong, J.; Zhang, L.; Zheng, J.; Liu, H.; Zhang, Q.; Ouyang, X.Q.; Lei, L.; Yu, X. Attenuation of inflammation by emodin in lipopolysaccharide-induced acute kidney injury via inhibition of toll-like receptor 2 signal pathway. Iran. J. Kidney Dis. 2015, 9, 202-208. [PubMed]

48. Zhang, F.; Wu, Z.J.; Sun, L.N.; Wang, J.; Tao, X.; Chen, W.S. Iridoid glucosides and a $\mathrm{C}_{13}$-norisoprenoid from Lamiophlomis. rotata and their effects on NF-kB activation. Bioorg. Med. Chem. Lett. 2012, 22, 4447-4452. [CrossRef] [PubMed]

49. Zhu, W.; Pang, M.; Dong, L.; Huang, X.; Wang, S.; Zhou, L. Anti-inflammatory and immunomodulatory effects of iridoid glycosides from Paederia scandens (LOUR.) MERRILL (Rubiaceae) on uric acid nephropathy rats. Life Sci. 2012, 91, 369-376. [CrossRef] [PubMed]

50. Hou, S.X.; Zhu, W.J.; Pang, M.Q.; Jeffry, J.; Zhou, L.L. Protective effect of iridoid glycosides from Paederia scandens (LOUR.) MERRILL (Rubiaceae) on uric acid nephropathy rats induced by yeast and potassium oxonate. Food Chem. Toxicol. 2014, 64, 57-64. [CrossRef] [PubMed]

(C) 2015 by the authors; licensee MDPI, Basel, Switzerland. This article is an open access article distributed under the terms and conditions of the Creative Commons by Attribution (CC-BY) license (http://creativecommons.org/licenses/by/4.0/). 